\title{
Characterisation of suprathermal electron pitch-angle distributions
}

\section{Bidirectional and isotropic periods in solar wind ${ }^{\star}$}

\author{
Fernando Carcaboso, Raúl Gómez-Herrero, Francisco Espinosa Lara, Miguel A. Hidalgo, \\ Ignacio Cernuda, and Javier Rodríguez-Pacheco
}

\begin{abstract}
Dpto. de Física y Matemáticas, Universidad de Alcalá, 28805 Alcalá de Henares, Madrid, Spain
e-mail: fernando.carcaboso@edu.uah.es
\end{abstract}

Received 26 August 2019 / Accepted 9 January 2020

\begin{abstract}
Context. Suprathermal electron pitch-angle distributions (PADs) contain substantial information about the magnetic topology of the solar wind. Their characterisation and quantification allow us to automatically identify periods showing certain characteristics. Aims. This work presents a robust automatic method for the identification and statistical study of two different types of PADs: bidirectional suprathermal electrons (BDE, often associated with closed magnetic structures) and isotropic (likely corresponding to solar-detached magnetic field lines or highly scattered electrons).

Methods. Spherical harmonics were fitted to the observed suprathermal PADs of the 119-193 eV energy channel of STEREO/SWEA from March 2007 to July 2014, and they were characterised using signal processing analysis in order to identify periods of isotropic and bidirectional PADs. The characterisation has been validated by comparing the results obtained here with those of previous studies.

Results. Interplanetary coronal mass ejections (ICMEs) present longer BDE periods inside the magnetic obstacles. A significant amount of BDE remain after the end of the ICME. Isotropic PADs are found in the sheath of the ICMEs, and at the post-ICME region likely due to the erosion of the magnetic field lines. Both isotropy and BDE are solar-cycle dependent. The isotropy observed by STEREO shows a nearly annual periodicity, which requires further investigation. There is also a correspondence between the number of ICMEs observed and the percentage of time showing BDE.

Conclusions. A method to characterise PADs has been presented and applied to the automatic identification of two relevant distributions that are commonly observed in the solar wind, such as BDE and isotropy. Four catalogues (STEREO-A and STEREO-B for isotropic and BDE periods of at least $10 \mathrm{~min}$ ) based on this identification are provided for future applications.
\end{abstract}

Key words. Sun: heliosphere - Sun: coronal mass ejections (CMEs) - solar wind - methods: data analysis - methods: statistical methods: analytical

\section{Introduction}

Suprathermal electrons ( $>60 \mathrm{eV}$ at $1 \mathrm{au}$, Feldman et al. 1975) are continuously streaming from the solar corona (Viñas et al. 2000; Štverák et al. 2008; Che \& Goldstein 2014; Graham et al. 2017) and propagate through the heliosphere following the interplanetary magnetic field (IMF) lines with a small gyroradius $(<22 \mathrm{~km}$, for $1 \mathrm{keV}$ and $5 \mathrm{nT}$ ). Their physical properties are routinely measured by in situ instruments aboard spatial observatories. They are normally divided into two different populations based on the velocity distribution function (VDF): a narrow beam that follows the IMF line (strahl), and an isotropic flux (halo) surrounding them (and references therein Anderson et al. 2012). In their travel they undergo some processes, such as scattering or adiabatic focusing depending on the IMF conditions (Owens et al. 2008). Due to this, the strahl can be scattered to contribute to the halo population (becoming broader), and the halo can be focused to form part of the strahl. The importance of these processes depends on the energy range, the IMF conditions, and

^ BDE and isotropic catalogues for both STEREOs are only available at the CDS via anonymous ftp to cdsarc.u-strasbg.fr (130.79.128.5) or via http://cdsarc.u-strasbg.fr/viz-bin/ cat $/ \mathrm{J} / \mathrm{A}+\mathrm{A} / 635 / \mathrm{A} 79$ the heliocentric distance. Nevertheless, the inter-dependency of all these factors is a complex issue, and there are contradictory examples of strahl width becoming narrower or broader depending on the situation (e.g. Anderson et al. 2012; Pagel et al. 2007; Berčič et al. 2019; Fitzenreiter et al. 1998; Horaites et al. 2018; Hammond et al. 1996; Graham et al. 2017). In order to identify the IMF topology among other physical conditions, it is crucial to accurately characterise the different types of suprathermal solar wind electron pitch-angle distribution (PAD).

The behaviour of the suprathermal electrons adds substantial information about the topology of the IMF lines and the connectivity between the Sun and the observer, as it acts as a relatively fast tracer of the IMF lines, due to their high velocity compared to that of the bulk (Owens \& Forsyth 2013). The study of the behaviour of the suprathermal electrons (e.g. strahl-halo evolution) has been traditionally performed by using moments of the electron VDFs derived on ground (e.g. Feldman et al. 1975). Also, one of the most valued sources of suprathermal electron IMF information is the analysis of the time evolution of the PAD, usually visualised as colour-coded plots (e.g. see panel 1 of Fig. 9). As the suprathermal electrons are streaming from the Sun (which adds a reference about the directionality), the shape of the PAD provides information of the in situ topology and acts 

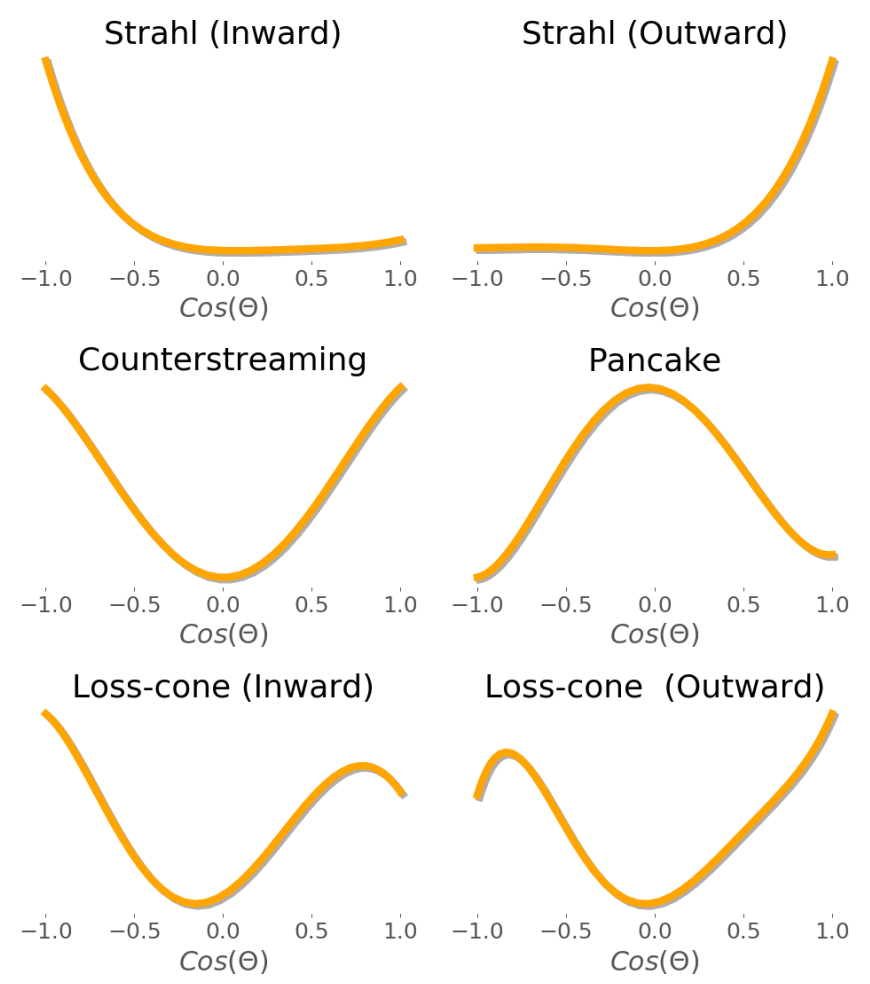

Isotropic

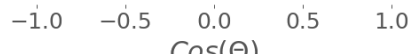

Fig. 1. Sketches of suprathermal electrons PAD patterns observed in the interplanetary medium. The horizontal axis represents the cosine of the pitch angle, while the vertical axis shows the VDF in linear scale.

as a complement for understanding the basics of the propagation of the suprathermal electrons. One of the direct applications of the information taken from the study of suprathermal electron PADs could be the computation of the heliospheric open solar flux (Owens \& Crooker 2006; Owens et al. 2013), or unravelling the IMF topology, among others (Kasper et al. 2019; Li et al. 2016). These PAD time series are also frequently used to provide largescale context for single spacecraft $(\mathrm{s} / \mathrm{c})$ observations of ICMEs (e.g. Nieves-Chinchilla et al. 2011). There are several commonly observed types of PADs of particular interest (sketched in Fig. 1):

- A simple strahl, which can be identified as a PAD clearly peaking either at $0^{\circ}$ or $180^{\circ}$. Assuming that there are no kinks in the field lines (causing an apparent sunward propagating strahl case, Owens et al. 2013, 2017), a VDF peaking at $0^{\circ}$ would correspond to positive (outward) IMF polarity, while if it peaks at $180^{\circ}$ the IMF line would have negative (inward) polarity. Moreover, the width of the strahl contains information about the interplanetary scattering process (Berčič et al. 2019; Maksimovic et al. 2005). In situ observations beyond 1 au suggest that the scattering from the strahl to the halo decreases with heliocentric distance and the strahl still exists up to at least 10 au (Hammond et al. 1996; Owens et al. 2008; Walsh et al. 2013; Graham et al. 2018).

- Counterstreaming (Gosling et al. 1987) appears when the PAD shows two clear maxima at $0^{\circ}$ and $180^{\circ}$. This can be the result of a mixture of two different beams coming along and against the IMF lines (i.e. a double strahl). As it results from a double stream, it is also commonly associated with a non-open IMF line (coming out from and back to the Sun).

- Loss-cone (Phillips et al. 1996). This phenomenon is produced when a simple strahl is being reflected from beyond the location of the observer due to a constriction of the IMF lines (acting as a magnetic mirror). The field-aligned electrons can pass through the constriction region, while those with larger pitch angles are mirrored back.

- Pancake (Kajdič et al. 2014). This distribution is symmetric with the absolute maximum at $90^{\circ}$ (inverted parabola) associated for instance by a betatron acceleration, produced by the reconnection of the magnetic field (see e.g. Liu et al. 2017; Wu et al. 2013, and references therein).

- Isotropic flux. Associated either with intense scattering (Gurgiolo \& Goldstein 2017), which smears the PAD, or with IMF lines detached from the Sun. (Wang et al. 2018). Heat flux dropout (HFD) events are intervals characterised by nearly isotropic suprathermal electron PADs (McComas et al. 1989; Crooker et al. 2003; Pagel et al. 2005a,b; Chollet et al. 2010), often found close to heliospheric current sheet (HCS) crossings. As suprathermal electrons are continuously streaming from the Sun, the lack of strahl and the isotropic PAD is interpreted either as an indication of magnetic structures disconnected from the Sun (via reconnection) or as a consequence of strong interplanetary scattering (see Pagel et al. 2005a, and references therein).

The PAD types listed above are examples of distributions commonly observed in the solar wind (SW), but they do not cover all the possible PADs that can be measured. Moreover, in some situations there could be more than one explanation for their existence in the SW.

Another commonly used term is bidirectional suprathermal electrons (BDE), which normally refers to the presence of beams propagating in both the field and anti-field aligned direction. Possible mechanisms for this behaviour are the double strahl, the overlap of a simple strahl and its reflection (due to e.g. converging IMF lines), or both adiabatic focusing and mirroring. This would include not only counterstreaming, but also losscone PADs, or even $90^{\circ}$ depletions (Gosling et al. 2001).

The presence of BDE is often an indicator of crossing an interplanetary coronal mass ejection (ICME, Montgomery et al. 1974; Bothmer et al. 1996; Gosling et al. 1987). ICMEs are the interplanetary counterpart of coronal mass ejections (CMEs), a phenomenon that consists of the release of large amounts of plasma from the solar corona, which often occurs at the same time as solar flares as a consequence of the reorganisation of the coronal magnetic field.

The presence of BDE inside ICMEs is often interpreted as an indication that these structures remain magnetically connected to the Sun at both ends (Feldman et al. 1999). Apart from the BDE, there is other evidence of this link to the Sun, such as the directionality of solar energetic particles (see e.g. Rodríguez-Pacheco et al. 2003; Gómez-Herrero et al. 2017). ICMEs are also identified by other signatures such as low plasma $\beta$, due to an enhanced IMF together with low proton temperature and density, smooth IMF rotations, decreasing SW speed (Zurbuchen \& Richardson 2006).

Interplanetary coronal mass ejections must eventually disconnect from the Sun, otherwise a continuous magnetic flux build-up would take place in the heliosphere (magnetic flux catastrophe, Gosling 1975; McComas 1995). The first proposed solution was the existence of reconnection processes that eventually produce a full disconnection of the magnetic structure at both ends; however, the evidence in support of this process 
(e.g. isotropic suprathermal electron PADs) is not frequent enough to account for the magnetic flux balance problem (McComas et al. 1989; Crooker et al. 2002). Another possibility is interchange reconnection with open field lines at one of the ICME legs close to the Sun or at some part of the structure in the interplanetary medium, leaving only one end of the ICME connected to the Sun. The interaction between ICMEs and the surrounding plasma in the corona or the SW implies a gradual erosion via interchange reconnection of the originally closed IMF lines, producing a mixture of open and closed field lines at $1 \mathrm{au}$; this is why the BDE intervals often do not cover the entire transit of the interplanetary structure, but have a "patchy" behaviour, with intervals showing other PAD shapes such as a simple strahl in the case of interchange reconnection (Larson et al. 1997; Crooker et al. 2002; Winslow et al. 2016; Ruffenach et al. 2015), or isotropy in the case of disconnection (Feng et al. 2018).

Therefore, the study of suprathermal PADs, especially the identification and characterisation of periods showing BDE, is crucial for the understanding of the magnetic topology of closed structures and their interaction with the surrounding medium, in particular the erosion processes of ICMEs. This work presents a new approach to the characterisation of PADs, with particular emphasis on BDE and isotropic periods, and is structured as follows. Section 2 presents the instrumentation and different catalogues used for the analysis. The observations and data analysis are presented in Sect. 3. The method developed for the characterisation of PADs is explained in Sect. 3.1. This method is applied to the study of anisotropy (Sect. 3.2.1) and to BDE (Sect. 3.2.2) in the SW observed by the Solar Terrestrial Relations Observatory (STEREO, Kaiser et al. 2008) during 20072014. After that, the ICMEs observed by the mission on the same period are studied from the point of view of these two classes of PAD (Sect. 3.3). A long-term analysis of the variation of the two PADs under study is presented in Sect. 3.4. An automatic process has been developed in order to produce catalogues of BDE and isotropic periods, and the results are shown in Sect. 3.5. Finally, a discussion and the conclusions are presented in Sect. 4.

\section{Instrumentation and datasets}

This work is based on the analysis of different in situ datasets from the STEREO mission. STEREO consists of two nearly identical s/c that travel approximately along the Earth's orbit and move away from the planet $\sim 22^{\circ}$ per year in opposite directions. Both s/c carry a comprehensive set of remote-sensing and in situ instruments. The in situ payload includes the Plasma and Suprathermal Ion Composition (PLASTIC, Galvin et al. 2008) instrument and the In situ Measurements of Particles and CME Transients (IMPACT, Luhmann et al. 2008) instrument suite. STEREO was launched in October 2006 and the data sample analysed in this work covers from March 2007 (when both s/c were outside the Earth's magnetosphere) until July 2014 (just before the superior solar conjunction started to affect the communications with ground). This period covers the solar minimum between cycles 23 and 24, and the rising phase of solar cycle 24 .

PLASTIC provides measurements of different plasma properties such as the bulk velocity, proton density, and temperature with a maximum resolution of one measurement per minute. IMPACT is a suite composed of multiple instruments. The ones used in this study are IMPACT-MAG (Acuña et al. 2008), which provides different components of the IMF strength with an acquisition frequency up to $32 \mathrm{~Hz}$ in burst mode, and the Solar Wind Electron Analyser (SWEA, Sauvaud et al. 2008). SWEA is able to measure the electron VDFs from $\sim 1 \mathrm{eV}$ to $3 \mathrm{keV}$. The instrument covers $360 \times 120^{\circ}$, and has a geometrical factor at $0^{\circ}$ of $8.4 \times 10^{-3}$ [ $\mathrm{sr} \mathrm{eV} \mathrm{cm}^{2} \mathrm{eV}^{-1}$ ], with a time resolution of approximately 2 counts $\mathrm{min}^{-1}$.

The SWEA scientific team provides the PAD of different energy channels as Level 2 data $^{1}$, with 12 equiangular calculated data points for every sample. The energy channel selected for study in this work ranges from $\sim 119 \mathrm{eV}$ to $\sim 193 \mathrm{eV}$, far from the core-suprathermal breakpoint, which can be found at around $60 \mathrm{eV}$ at $1 \mathrm{au}$ (Feldman et al. 1975). During the period from 14 February 2008 to 16 April 2009 for STEREO-A, and from 2008-02-16 to 2009-04-16 for STEREO-B, the PAD data product covers a slightly different energy range from $\sim 127 \mathrm{eV}$ to $\sim 180 \mathrm{eV}$. It should be noted that the study presented here focuses on the shape of the PAD, for this reason the methods discussed in Sect. 3.1 are not significantly affected by instrument gain changes.

Apart from the STEREO in situ data, a selection of ICMEs from the ICME STEREO/MAG list maintained by L. Jian (hereafter ICME list) ${ }^{2}$ were considered for the statistical analysis. The selection criteria of the ICMEs can be found in the series of publications Jian et al. (2018, 2013, 2006a). The Stream Interaction Region (SIR) STEREO/MAG list maintained also by L. Jian (hereafter SIR list) ${ }^{3}$ was used to identify the quiet periods of the SW (see Sect. 3.2.2). The selection criteria and further studies of the catalogued SIRs are covered by Jian et al. (2019, 2006b). Finally, in order to have a broader overview of the context of the interplanetary medium for the statistical analysis and also to define the quiet SW, the Heliospheric Shock Waves Database maintained by the University of Helsinki (hereafter shock list) ${ }^{4}$ is used in this study.

\section{Observations and data analysis}

Pitch-angle distribution signatures are often determined by eye using 3D colour-coded plots of PAD time evolution (e.g. Shodhan et al. 2000); however, they can also be characterised numerically (see e.g. Chen et al. 2014). This is introduced in Sect. 3.1. A numeric characterisation can be automatised and therefore provides a better approach for statistical studies. When the value of the distribution is higher close to the edges of the $\operatorname{PAD}\left(0^{\circ}\right.$ and $\left.180^{\circ}\right)$ than at the centre $\left(90^{\circ}\right)$, BDE may be present. If the intensity is higher at $\sim 90^{\circ}$, then a pancake distribution may be present. Moreover, when the intensity is similar at all pitch angles, it is considered an isotropic flux. On the other hand, if the intensity is higher close to $0^{\circ}$, a strahl is present along outward IMF, while if the intensity is higher close to $180^{\circ}$, the IMF would be inward (unless sunward propagating strahl case, Owens et al. 2013).

Figure 2 shows an example of STEREO-B in situ observations during 6-11 May 2014. This interval includes a period showing BDE during 8, 9, and 10 May 2014 (days of year 128, 129 , and 130 respectively), also corresponding to the transit of an ICME. This ICME is preceded by a simple strahl along an outward IMF and followed by another strahl along the inward IMF. The vertical dashed green line marks an interplanetary shock

\footnotetext{
1 https://stereo-ssc.nascom.nasa.gov/data/ins_data/ impact/level2

2 https://stereodata.nascom.nasa.gov/pub/ins_data/ impact/level3/STERE0_Level3_ICME.pdf

3 https://stereodata.nascom.nasa.gov/pub/ins_data/ impact/level3/STEREO_Level3_SIR.pdf

4 http://ipshocks.fi
} 


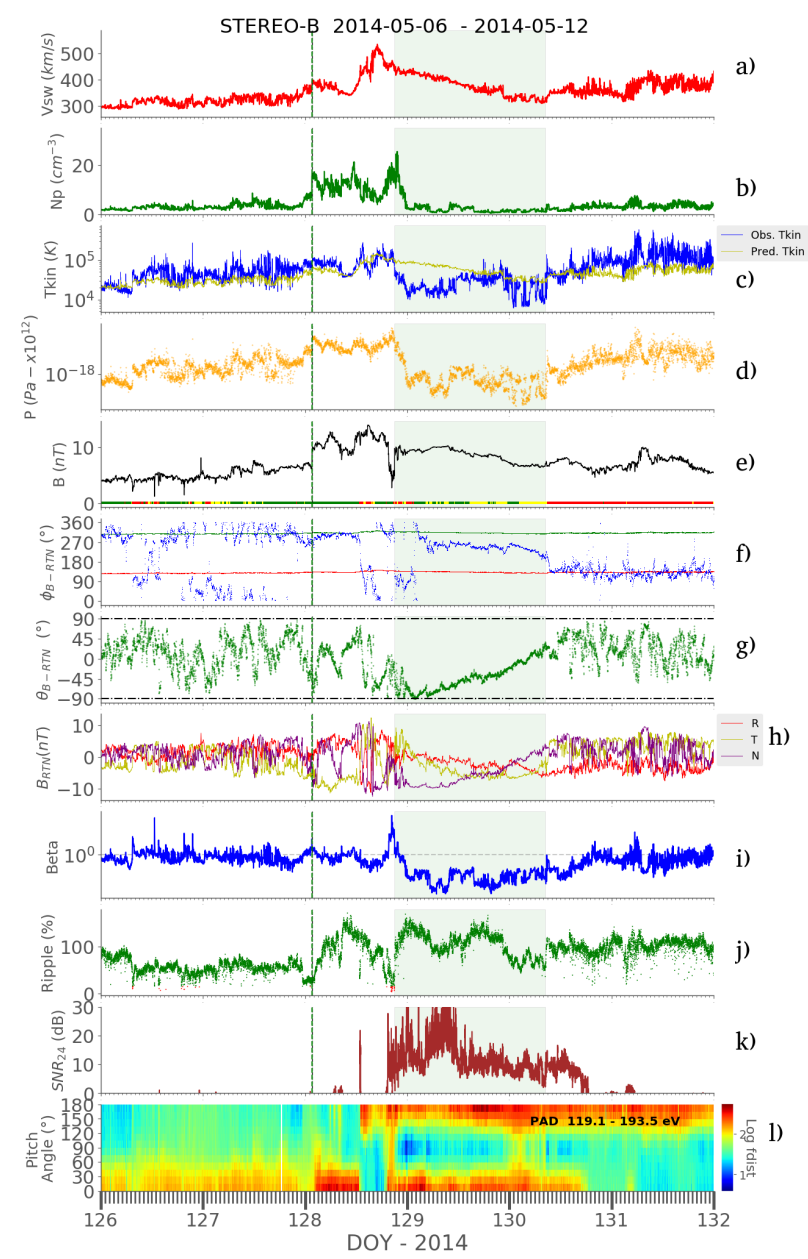

Fig. 2. Period showing BDE observed by STEREO-B on May 2014 during the transit of an ICME. From top to bottom: SW proton speed, proton density, proton temperature, IMF magnitude accompanied by its polarity (red, negative; green, positive; yellow, ambiguous), IMF azimuthal angle in the RTN coordinate system complemented with the two possible nominal Parker spiral angles (red, negative; green, positive) calculated from the proton speed, IMF latitudinal angle in the RTN coordinate system, RTN magnetic field separated components, plasma $\beta, \gamma$ (ripple; see Sect. 3.1), $S / N_{24}$ (see Sect. 3.1), and 119$194 \mathrm{eV}$ suprathermal electron PAD colour-mesh $(y$-axis is the angle in degrees, $z$-axis the VDF in logarithmic scale). The vertical green dashed line indicates the interplanetary shock catalogued in the Shock list, the green shaded area corresponds to the magnetic obstacle as stated in the ICME list.

(catalogued by the shock list, which also matches the ICME list), and the light green shaded area indicates the magnetic obstacle (MO, interpreted as the magnetic structure intrinsic to the ICME, but not necessarily manifesting a flux rope topology) corresponding to the ICME catalogued at the ICME list. In panel c the yellow line represents an empirically predicted kinetic temperature based on the proton speed Elliott et al. (2012). When the actual proton temperature is lower than the calculated (predicted), it could indicate the presence of an MO as the structure is colder than expected due to the adiabatic cooling. In panel e the colour bars show the polarity of the IMF observations, assuming a nominal Parker spiral angle based on the SW velocity with a range covering $\pm 60^{\circ}$ (red: negative or inward; green: positive or outward; yellow: out of the nominal Parker spiral). In plot $f$ the green and red lines correspond to that nominal Parker spiral azimuthal IMF angle (positive and negative, respectively), while plot $g$ represents the latitudinal angle. Panel h shows the three different components of the IMF in RTN coordinates. Graph $i$ shows the plasma $\beta$, calculated as explained in Sect. 3.2.1. As stated in Sect. 1, the MO is characterised by a low proton temperature, smooth magnetic field rotations, low plasma $\beta$, decreasing SW speed profile and BDE. Panels $\mathrm{j}$ and $\mathrm{k}$ show the anisotropy index $\gamma$ and the $S / N_{24}$ of the suprathermal electrons PAD (see following Sect. 3.1).

\subsection{Pitch angle characterisation}

Under the assumption of a gyrotropic trajectory, the suprathermal electron PADs $(f(\theta)$, Eq. (1)) can be characterised using an expansion of the orthogonal Legendre polynomials on the cosine of the pitch angle

$f(\theta)=\sum_{i=0}^{\infty} A_{i} P_{i}(\cos \theta)=\sum_{i=0}^{\infty} F_{i}(\theta)$,

where $\theta$ represents the pitch angle, $A_{i}$ are numeric coefficients, and $P_{i}(x)$ are the Legendre polynomials, given by Rodrigues formula (e.g. Howlett et al. 2007):

$P_{i}(x)=\frac{1}{2^{i}} \sum_{k=0}^{i}\left(\begin{array}{l}i \\ k\end{array}\right)^{2}(x+1)^{i-k}(x-1)^{k}$.

A similar approach has been used to analyse the PAD of solar energetic particles (Balogh 1971; Sanderson et al. 1983, 1985; Agueda \& Lario 2016) and energetic electrons in the radiation belts surrounding Earth (Chen et al. 2014). Legendre polynomials formally include infinite harmonics $(i \rightarrow \infty)$; however, the experimental data constitutes a discretisation which limits the maximum meaningful order. The process followed in this work is to fit a certain number of Legendre polynomials to all PAD data with total IMF coverage, and to correct for ion bulk flow by using a least-squares fitting algorithm denominated LevenbergMarquardt under the Python 3.6 Imfit package (Newville et al. 2014, 2019). Previous methods (such as the Gaussian fitting used in e.g. Graham et al. 2018 or Anderson et al. 2012) ensure that the fittings only include clear strahl signatures, while this method, as in Chen et al. (2014), covers all PAD shapes excluding only those fits not converging. This happened for only a very small minority of total number of fits. Due to the leastsquares fitting, artificial oscillations at the boundaries of the studied interval may occur. This problem is known as Runge's phenomenon and occurs when a polynomial is fitted to a set of equispaced data points. For this reason, it is necessary to have a well-conditioned approximation (Dahlquist \& Björck 2014), and the order of the harmonics must accomplish $i<2 \sqrt{n}$ (where $n$ is the number of data points) if a least-squares fitting is performed. As seen in the previous Sect. 2, the SWEA PAD Level2 dataset provides 12 equiangular data points for every sample. In this case the selected harmonics (truncating Eq. (1)) go up to the fifth order:

$F_{0}(\theta)=A_{0}$

$F_{1}(\theta)=A_{1} \cos (\theta)$

$F_{2}(\theta)=A_{2} \frac{1}{2}\left(3 \cos ^{2}(\theta)-1\right)$

$F_{3}(\theta)=A_{3} \frac{1}{2}\left(5 \cos ^{3}(\theta)-3 \cos (\theta)\right)$

$F_{4}(\theta)=A_{4} \frac{1}{8}\left(35 \cos ^{4}(\theta)-30 \cos ^{2}(\theta)+3\right)$ 


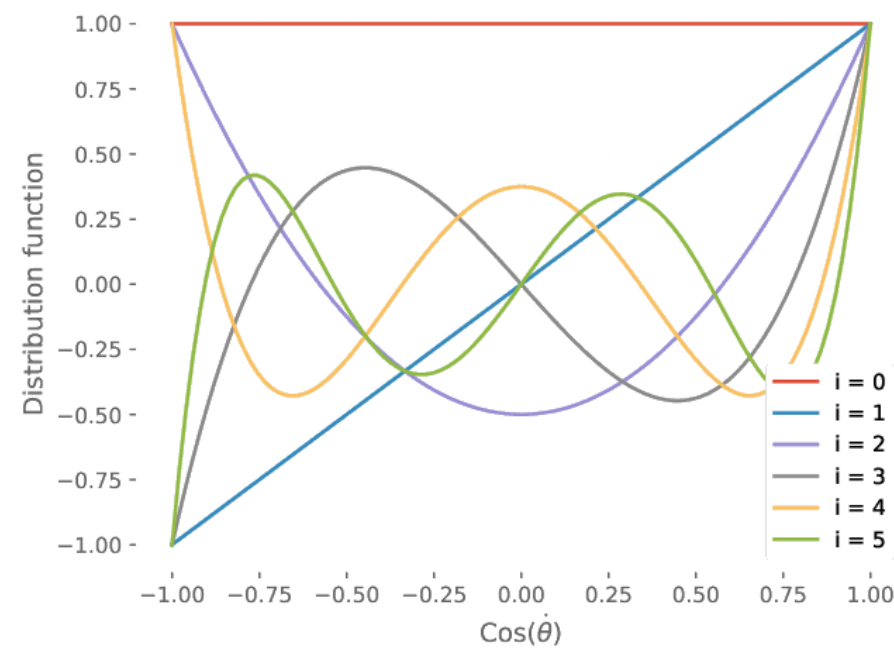

Fig. 3. Legendre Polynomials up to the fifth order. The summation of all harmonics, multiplied by a coefficient, reproduces the final PAD. Shown are $i=0$, which represents the mean value; the even harmonics $(2,4)$, which are symmetric along $\cos (\theta)=0$; and the odd harmonics $(1,3,5)$, which are antisymmetric.

$F_{5}(\theta)=A_{5} \frac{1}{8}\left(63 \cos ^{5}(\theta)-70 \cos ^{3}(\theta)+15 \cos (\theta)\right)$.

The shape of these six terms (for $A_{i}=1$ ) are shown in Fig. 3. The coefficients $A_{i}$ are obtained by fitting the summation of the six terms (from Eqs. (3)-(8)) to the experimental PAD data points. Some examples of the performed fits can be found in Fig. 4.

Even Legendre polynomial terms $\left(F_{2}, F_{4}\right)$ show a symmetric distribution (with respect to $90^{\circ}$, or $\cos (\theta)=0$ ), while the odd ones $\left(F_{1}, F_{3}, F_{5}\right)$ have antisymmetric behaviour, and $F_{0}$ corresponds to the mean value of the PAD. The global shape of the PAD is determined by the relative contribution of each harmonic:

- Bidirectional PADs can be considered to be symmetric flux as a first approximation. An ideal counterstreaming would appear when the second- and fourth-order coefficients are positive and have much higher absolute value than the others, while loss-cone PADs or $90^{\circ}$ depletions would also present symmetry, but their identification requires further interpretation of the coefficients.

- A pancake is also a symmetric PAD, but in contrast to the counterstreaming case the second coefficient must be negative and dominate over the others.

- When the mean value $\left(A_{0}\right)$ is the predominating contribution, then the PAD is isotropic.

In other words, these three PADs can all be interpreted as symmetric PADs, where the fit is dominated by the contribution of the even terms $A_{2}, A_{4}$ for BDE and pancake (with opposite signs), and by $A_{0}$ for isotropic flux.

The importance of the contribution of each term to the final DF can be characterised using a logarithmic relative power scale. Analogously to signal processing methods, we denote this defining a signal-to-noise-ratio $(\mathrm{S} / \mathrm{N})$, taking the harmonic(s) under study as signal, and the rest of the harmonics as the noise:

$S / N_{\mathrm{dB}}=10 \cdot \log _{10}\left(\frac{\mathcal{P}_{\text {signal }}}{\mathcal{P}_{\text {noise }}}\right)$.

Here $\mathcal{P}_{\text {signal }}$ and $\mathcal{P}_{\text {noise }}$ are the power of one or the summation of various harmonics. The power of any harmonic/s is defined as

$\mathcal{P}_{i}=\frac{1}{2 \pi} \int_{-\pi}^{\pi}\left|F_{i}(\theta)\right|^{2} \mathrm{~d} \theta$

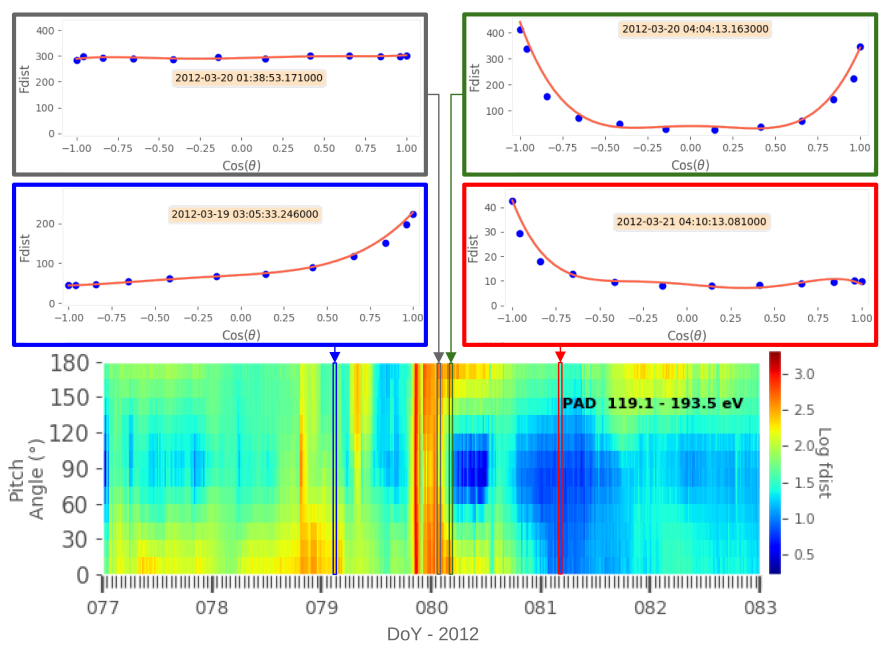

Fig. 4. Four different fits of STEREO-A observations during March 2012 accompanied by their timestamps, and colour-coded PAD as presented in panel $l$ of Fig. 2. Shown are isotropic flux (grey box), strahl with outward polarity (blue box), BDE distribution (green box), and strahl with inward polarity (red box).

As mentioned above, the second and the fourth coefficients are the ones that define the symmetry of the PAD. For this reason, the $\mathrm{S} / \mathrm{N}$ of the sum of the two harmonics $\left(S / N_{24}\right)$ has to be, at least, higher than $0 \mathrm{~dB}$ in order to present a symmetric flux (e.g. a bidirectional or a pancake VDF), and the higher this parameter is, the more symmetric the flux.

When the VDF is nearly isotropic, the possible contributions of the harmonics are negligible compared to the value of $A_{0}$ (mean value). For this reason, a ripple coefficient $\gamma$ can be defined in order to identify how significant the angle-dependent deviations are:

$\gamma=100 \cdot \frac{f_{\mathrm{rms}}}{A_{0}}[\%]$

where

$f_{\mathrm{rms}}=\sqrt{\frac{1}{2 \pi} \int_{-\pi}^{\pi}|f(\theta)|^{2} \mathrm{~d} \theta}$.

This coefficient characterises the existence of anisotropy. Perfect isotropy would correspond to $\gamma=0$, while large values characterise highly anisotropic VDFs.

The definition of a threshold in $\gamma$ allows an easy separation of isotropic and anisotropic periods ( $\gamma_{\text {th }}$; see Sect. 3.2.1), while the combined use of $S / N_{24}$ and $\gamma_{\text {th }}$ permits the identification of bidirectionality (see Sect. 3.2.2). The following subsections illustrate the application of these procedures to real STEREO/SWEA data. Every single 30-s sample of suprathermal electron PAD provided by STEREO/SWEA in the energy range $\sim 119 \mathrm{eV}$ to $\sim 193 \mathrm{eV}$ between March 2007 and July 2014 was fitted in order to obtain the Legendre polynomial coefficients $A_{i}$ for both s/c. Once they were derived, the $S / N_{24}$ and $\gamma$ were calculated. This information can be used for automatic characterisation of the PAD shape. The method presented in this work can be easily generalised to similar instruments (for instance ACE/SWEPAM) or to other particle fluxes with gyrotropic behaviour (e.g. solar energetic particles), and permits an automatic search and classification of suprathermal electron PADs over extended periods of time. 


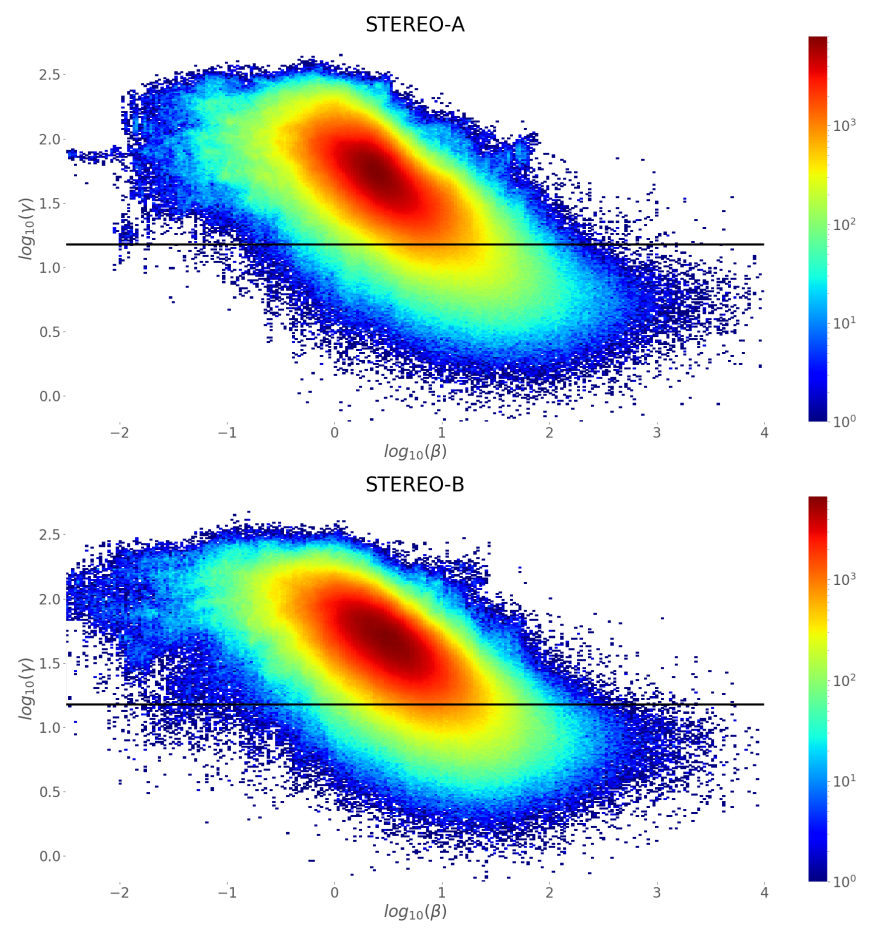

Fig. 5. Two-dimensional histograms showing the relation between the ripple $(\gamma)$ and the $\log _{10}$ of the plasma $\beta$ for the entire period under study (2007-2014) for STEREO-A (top) and STEREO-B (bottom). The horizontal black line shows $\gamma_{\mathrm{th}}$, and the colour bar indicates the number of events for each bin in logarithmic scale.

\subsection{Suprathermal electron pitch-angle distributions in the solar wind}

\subsubsection{Characterising anisotropy}

Plasma $\beta$ is defined as the ratio of the gas pressure (calculated as $P_{\mathrm{g}}=N_{\mathrm{p}} K T_{\mathrm{p}}+N_{\mathrm{e}} K T_{\mathrm{e}}+N_{\mathrm{He}} K T_{\mathrm{He}}$, where $N$ is the density, $K$ the Boltzmann constant, and $T$ the temperature) to the magnetic pressure. The magnetic pressure is calculated as $P_{\mathrm{mag}}=B^{2} / 2 \mu_{0}$ (with $B$ the magnetic field and $\mu_{0}$ the vacuum permeability), while the gas pressure is estimated as the pressure considering the contribution of protons, alphas, and electrons, as explained in Mullan \& Smith (2006), assuming $T_{\mathrm{e}}$ and an alpha-to-proton ratio constant with a value of $140000 \mathrm{~K}$ and 0.04 , respectively (Newbury 1996; Bürgi 1992).

It is well established that there is a correspondence between plasma $\beta$ and the anisotropy of suprathermal electrons in the SW (Crooker et al. 2003). The value of plasma $\beta$ is anticorrelated with the anisotropy. High-beta plasmas are prone to scattering suprathermal electrons, and this reduces heat flux coming from the Sun. In order to validate the characterisation of the degree of anisotropy based on the ripple coefficient $\gamma$, Fig. 5 shows a 2D histogram of $\gamma$ versus $\log _{10}(\beta)$ for the entire period under study for both STEREOs. In both cases, there is a clear decreasing trend of $\gamma$ (see also Fig. 4 in Crooker et al. 2003, where the authors characterise the anisotropy using the variance of the experimental PAD data). This confirms that $\gamma$ can be used as a reliable anisotropy index.

It should be noted that values of $\gamma$ are bounded between $\sim 1 \%\left(\log _{10}(\gamma)=0\right)$ and $\sim 320 \%\left(\log _{10}(\gamma)=2.5\right)$, and that the contribution of the harmonics to the final VDF is rarely higher than 3.2 times the value of $A_{0}$. For practical purposes and taking into account the existence of statistical fluctuations in the

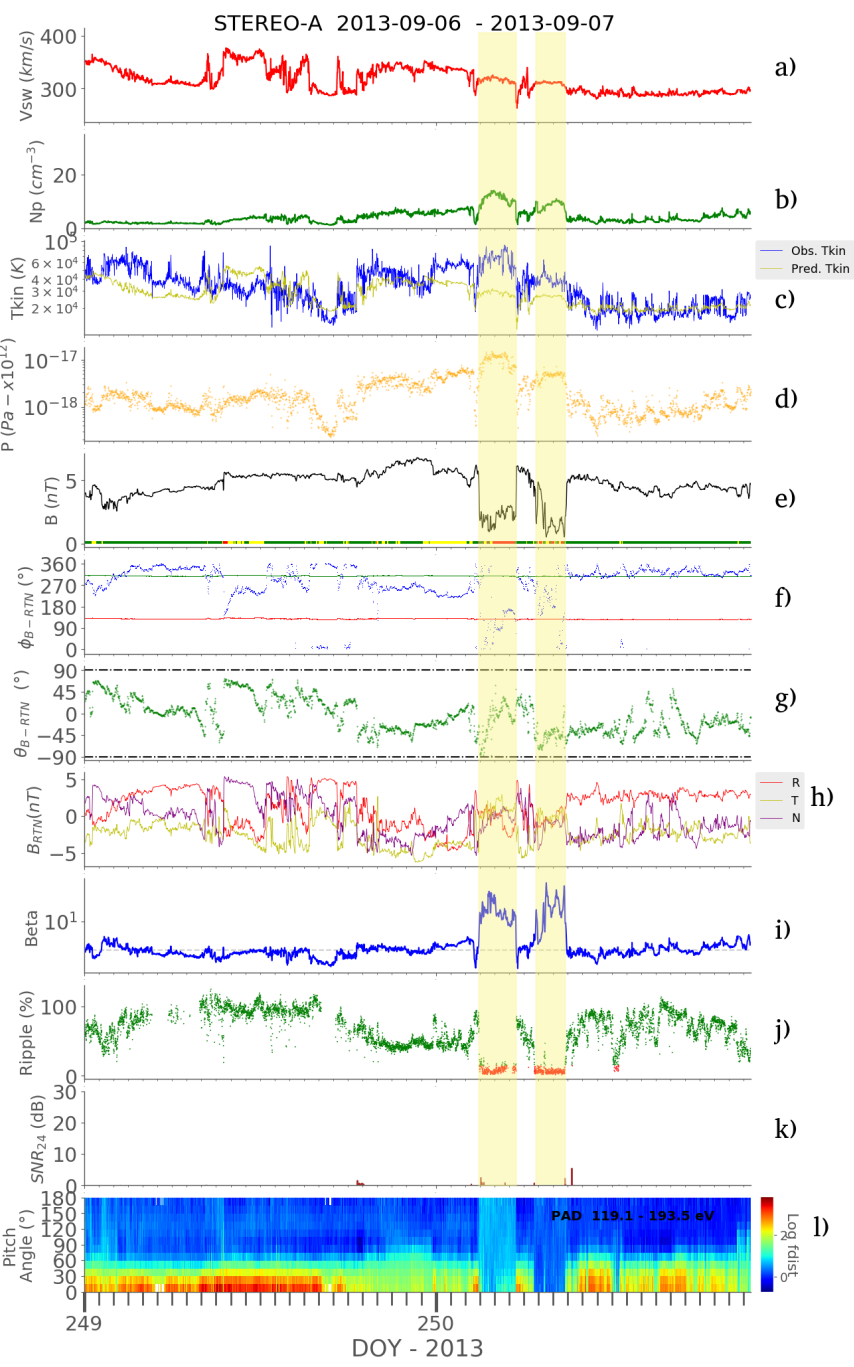

Fig. 6. STEREO-A observations during a period in September 2013 including two intervals of isotropic flux of suprathermal electrons shaded in yellow. The panel content follows the same format as in Fig. 2.

experimental data, an upper threshold value of $\gamma_{\text {th }}=15 \%$ is used as a selection criterion to tag isotropic periods (i.e. the total contribution of the harmonics should be at least $15 \%$ with respect to the mean value).

As an example, Fig. 6 shows a period including two closely spaced time intervals where the PAD becomes almost isotropic. Both intervals are shaded in yellow. These periods, with very sharp boundaries, also show clear signatures in different SW parameters such as IMF decrease, proton density enhancement with the corresponding increase in the plasma $\beta$. As can be seen, these periods are easily identifiable by the low values of the anisotropy index $\left(\gamma \leq \gamma_{\text {th }}\right.$, in red in panel $\mathrm{j}$ of Fig. 6). The rest of the shown interval is characterised by a strahl with outward polarity and higher values of $\gamma$.

Using a threshold value of $\gamma \leq \gamma_{\mathrm{th}}$, a complete survey of isotropic PADs was performed for the period under study. From this survey, a list of isotropic periods lasting for at least $10 \mathrm{~min}$ was compiled (see Sect. 3.5).

\subsubsection{Characterising bidirectionality}

In order to define a reliable criterion for the identification of BDE periods, observations of suprathermal electron PADs from 


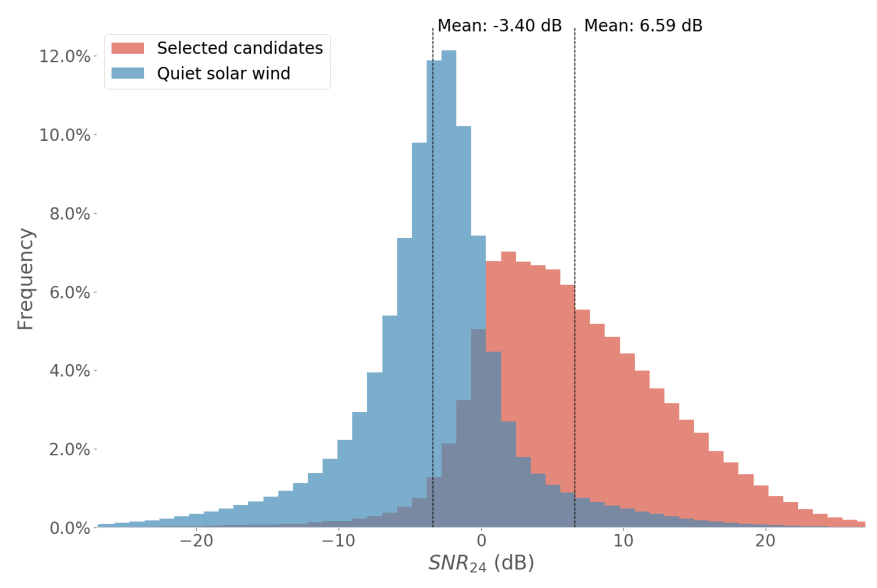

Fig. 7. Histograms of the calculated $S / N_{24}$ for the selection of ICMEs with longer periods of BDE (red, listed in Appendix A), and for quiet SW (blue).

$\sim 119 \mathrm{eV}$ to $\sim 193 \mathrm{eV}$ from ICMEs and from quiet SW are compared. A subset of ICMEs observed by both STEREOs with particularly long and clear periods of BDE was filtered by eye (Appendix A). After this selection, the data were filtered using the criteria of $\gamma>\gamma_{\text {th }}$ (i.e. non-isotropic) defined in Sect. 3.1 and $A_{2}>0$ (i.e. not peaking near pitch angle $90^{\circ}$ ). Once they were filtered, the $S / N_{24}$ was calculated. The same procedure was performed for the selection of quiet SW periods, defined as the whole period under study, removing the transit of the catalogued ICMEs $+12 \mathrm{~h}$, SIRs $\pm 12 \mathrm{~h}$, and shock $\pm 2 \mathrm{~h}$ (see Sect. 2).

Histograms of the calculated $S / N_{24}$ for each sample are presented in Fig. 7. For the quiet SW (blue), the distribution is almost symmetric, centred at a mean value of $S / N_{24}$ of $-3.40 \mathrm{~dB}$. As expected, the distribution for the selected ICMEs (red) is significantly shifted to higher values of $S / N_{24}$, showing an asymmetric distribution with a mean value of $6.59 \mathrm{~dB}$, and a median value of $5.79 \mathrm{~dB}$ of a total of 370875 fits. Both distributions intersect near $0 \mathrm{~dB}$ and show some overlapping due to the occasional presence of BDE in the quiet SW (e.g. due to noncatalogued ICMEs, small-scale transients or post-ICME periods showing significant bidirectionality; see Sect. 3.3) and the absence of BDE during certain periods of the selected ICMEs as well.

In order to set a reliable identification criterion of periods with clear BDE, a threshold value of $S / N_{24}$ corresponding to $50 \%$ of the transit of the selected ICMEs observing BDE periods was fixed (i.e. the median $S / N_{\text {th }}=5.79 \mathrm{~dB}$ ). This restrictive threshold does not imply that the values below do not correspond to bidirectional VDFs, but it assures that those above $5.79 \mathrm{~dB}$ are almost certainly bidirectional. The stated threshold $\left(S / N_{\mathrm{th}}\right)$ is used in following the studies in Sects. 3.3-3.5. It should be noted that this selection threshold is always used for relative comparisons of different periods, and not as an absolute indicator of BDE.

\subsection{Suprathermal electrons in ICMEs}

\subsubsection{Bidirectionality}

During the transit of an ICME, s/c sometimes observe noncontinuous or even non-existent periods of BDE (Larson et al. 1997; Winslow et al. 2016). The BDE is mainly produced by the stream of suprathermal electrons from both foot-points of the ICME. As stated in Sect. 1, magnetically closed structures can

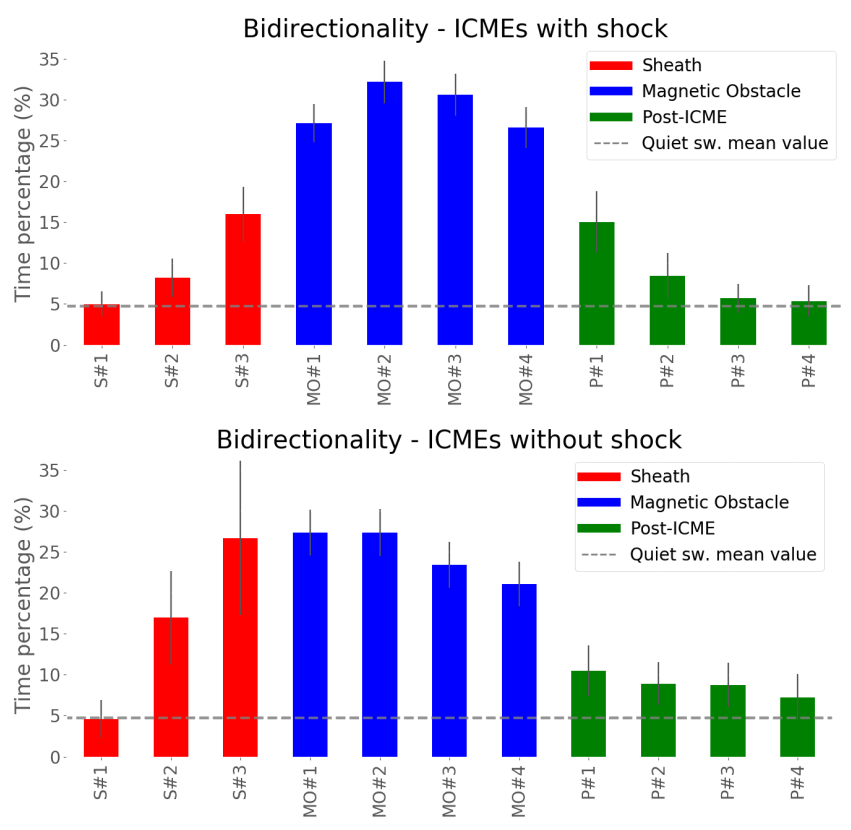

Fig. 8. Histograms showing the mean value of the time-percentage of BDE for each sector during all the ICMEs transits catalogued in the ICME list, with shock at the beginning (top) and without (bottom). The colours show the different catalogued regions (see text for more details) and the labels correspond to equi-timed periods of the transit of those regions ( $\mathrm{S}$ - sheath, $\mathrm{MO}$ - magnetic obstacle, $\mathrm{P}$ - post-ICME). The error bars indicate the standard error of the mean.

be gradually eroded by reconnection processes, with a consequent loss of BDE.

In order to study how $\mathrm{BDE}$ is distributed during the transit of ICMEs, and in the immediately trailing region, a superposed epoch analysis was performed, considering all the ICMEs catalogued on the ICME list observed by both STEREOs during the period under study (317 ICMEs in total, excluding those affected by data gaps). The superposed epoch analysis was performed by dividing the ICME time-transit into different sections, and the time-percentage mean value of BDE (where $S / N_{24}$ is higher than the previously defined threshold $S / N_{\text {th }}$ ) was calculated for each of them. Later on, the average of all the mean values in each section was obtained. The MO was divided into four parts, while the sheath (if present), due to the lack of statistics because it is shorter, was divided into thirds. The interval following the ICMEs (hereafter post-ICME) was studied, excluding those cases where any other catalogued structure (ICME, SIR, or interplanetary shock) is present. The duration of this final region for each event was set as 1.2 times the total duration of each ICME, and was divided into four chunks. This is the selected value because it corresponds to the minimum time required to recover the quiet SW time-percentage mean value of BDE for the whole sample of ICMEs (not shown). Figure 8 shows cumulative (using all the catalogued ICMEs) histograms of the timepercentage mean value of BDE (calculated using the criteria explained in Sect. 3.2.2). The ICMEs have been classified into two different types: with and without shocks (top and bottom histograms in Fig. 8, respectively) based on the catalogues used throughout the study. The horizontal dashed line corresponds to the time-percentage mean value observed in the quiet SW, as defined in previous Sect. 3.2.2.

The average duration of the sheath region is $9.9 \pm 0.5 \mathrm{~h}$ (15 events for ICMEs without shock, and 67 for ICMEs with shock), while the MO has a mean duration of $24.3 \pm 0.8 \mathrm{~h}$ 
(139 events for ICMEs without shock, and 178 for ICMEs with shock). Finally, the post-ICME region lasts for $34.6 \pm 2.0 \mathrm{~h} \mathrm{(45}$ events for ICMEs without shock, and 42 for ICMEs with shock). We note that post-ICMEs are not considered if another catalogued event overlaps.

Based on the results shown in Fig. 8, there is a clear tendency for both types of ICMEs (with and without shock) to have a gradual increase in the time presenting bidirectionality until the end of the first half of the MO, where it starts to decrease. As shown in Feldman et al. (1999), among others, there is a tendency of BDE present inside of the ICMEs to be higher inside the MOs. The amount of BDE time-percentage becomes maximum in the central part of the MO, which is more shielded from the surrounding fields. The presence of BDE inside the sheath is a likely indication of the existence of some closed loops in that region.

In the case of ICMEs with shock, the MO presents longer periods of BDE and the difference between the amount of BDE inside the MOs and the sheath and post-ICME is clearer than in the case of ICMEs without shocks. A possible explanation is that those events driving an interplanetary shock constitute a subset of the fastest ICMEs, which consequently have less time for undergoing erosion during the interplanetary propagation to 1 au.

On the other hand, ICMEs without shock do not show a symmetric profile of the percentage of BDE inside the MO, as expected considering that they are composed of flux-ropes and the IMF lines crossed by the s/c are the same at the front and the rear part. In contrast, the first half of the MO shows more BDE than the second. This could be an indication of stronger erosion in the trailing edge of the expanding MO.

In both cases it is notable that the post-ICME contains periods with longer bidirectionality than the quiet $\mathrm{SW}$, especially for the ICMEs without shocks, and that it decreases gradually. The existence of BDE at the post-ICME indicates the presence of either structures or special IMF conditions after MOs. Some of the multiple explanations to this may be the existence of isolated closed lines in post-ICME or the presence of uncatalogued flux ropes (in the ICME list) after the ones studied. Another explanation could be the reflection of strahl occurring in converging IMF lines around the ICME (or at the shock, if it exists).

As an illustrative example, Fig. 9 shows an ICME with behaviour resembling the average profile of BDE timepercentage previously shown in Fig. 8, for the MO and the postICME period. The ICME is preceded by a simple strahl with inward IMF and followed by $\sim 1.5$ days of BDE, and accompanied by a noticeable shock. The BDE during the MO and the post-ICME period are easily identified by the enhanced values of the $S / N_{24}$ (panel k). As previously seen in Sect. 3, panel j shows the ripple $\gamma$. Periods corresponding to $\gamma \leq \gamma_{\text {th }}$ are in red (flagged as isotropic periods). In the plot, this corresponds to the midday of DoY 276 and during the sheath region of the ICME. Isotropy during and after ICMEs are discussed in the following section.

\subsubsection{Isotropy}

Similarly to Sect. 3.3.1, a study of the time-percentage of isotropy inside ICMEs and during the post-ICME region was performed.

Figure 10 shows the same superposed-epoch sample of ICMEs presented in Fig. 8, but in this case representing the fraction of time showing isotropy, defined as $\gamma \leq \gamma_{\mathrm{th}}$. The grey dashed line marks the average percentage of isotropy observed in

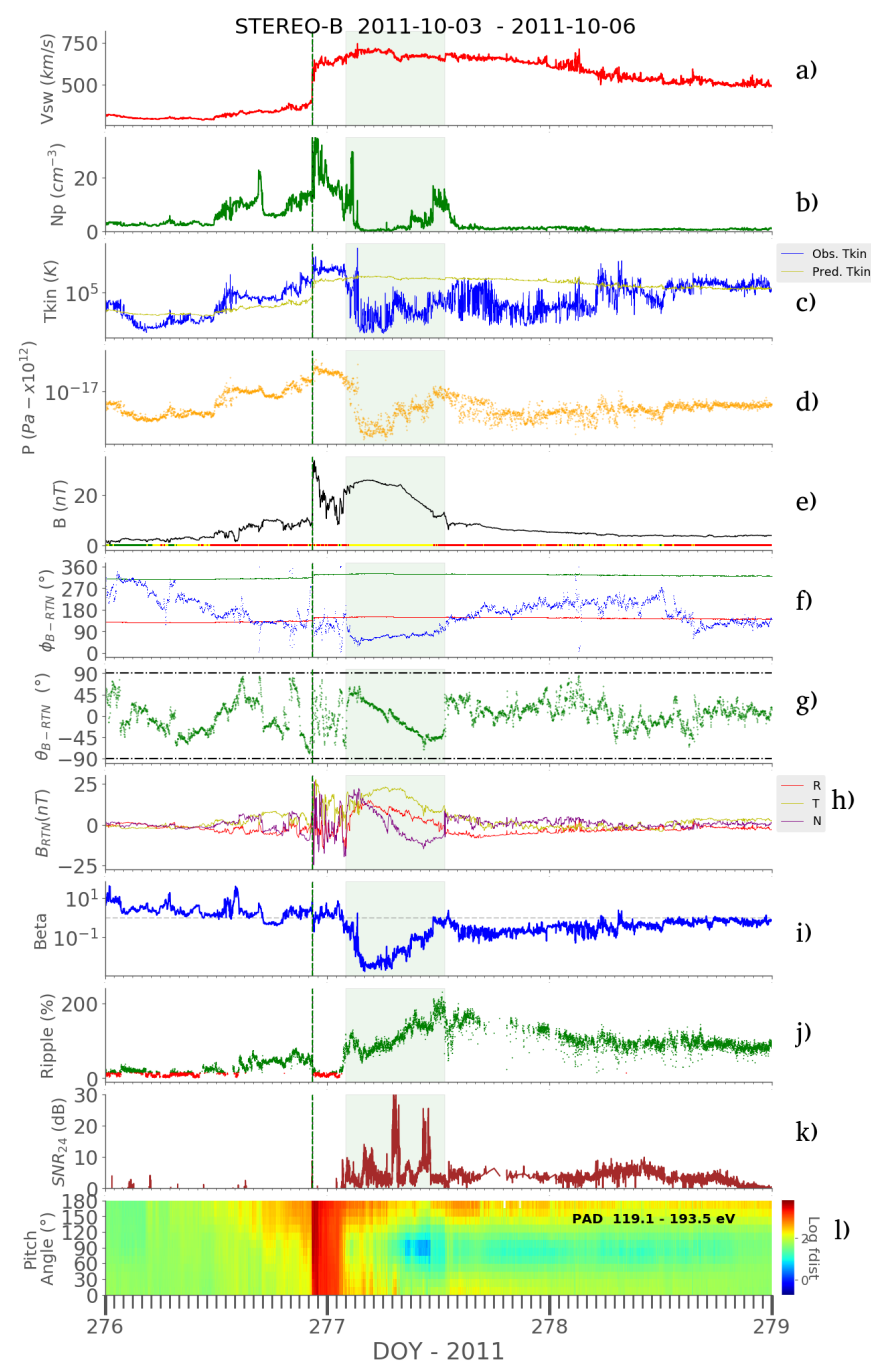

Fig. 9. STEREO-A observations during a period in October 2011 with one of the studied ICMEs. The panel content follows the same format as in Fig. 2.

the quiet SW (as defined in Sect. 3.2.2). The MO (blue) presents the lowest probability of presenting isotropy, always below the average isotropy in the quiet $\mathrm{SW}$, while the sheath (red) and the post-ICME (green) have higher values. Also, ICMEs with shock show a higher rate of isotropy in the sheath. Since the sheath is a compressed turbulent region (see e.g. Kilpua et al. 2017), this isotropy could be partly due to enhanced scattering conditions. The enhanced isotropy in the post-ICME region (clearer for the ICMEs without shock) could be an indication of the presence of formerly closed field lines that became fully disconnected from the Sun at both ends.

The ICME event shown in Fig. 9 is a good example of the existence of isotropy in the sheath region. Figure 11 shows one of the studied ICMEs observed by STEREO-B during solar maximum (October 2013), which clearly shows isotropy in the postICME region. That ICME is accompanied by a shock (vertical green dashed line) catalogued in Shock list and presents clear signatures such as decreasing SW speed, enhanced IMF, low plasma $\beta$, low proton temperature, and BDE. The MO is shaded in green. The sheath is between the shock and the MO, and the post-ICME lasts until the end of 12 October (DoY 285). Panel j shows the anisotropy index $\gamma$, and turns red when $\gamma \leq \gamma_{\text {th }}$ (i.e. corresponding to isotropic periods). 


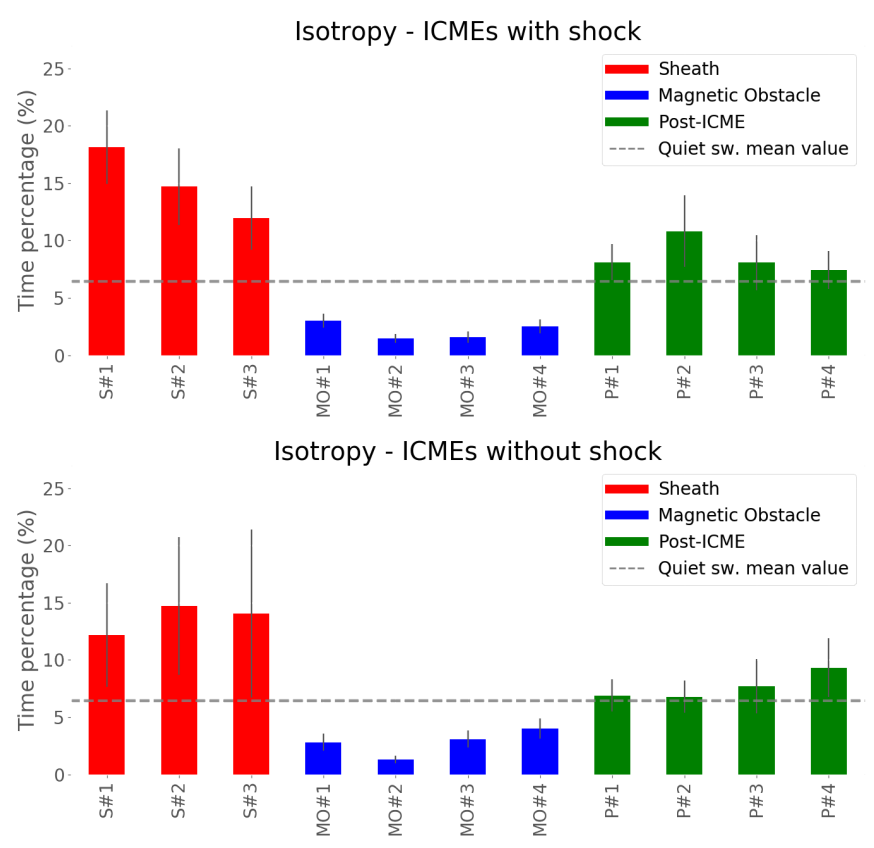

Fig. 10. Histograms of mean value of the time-percentage of isotropy of each sector during all the ICMEs transits catalogued in the ICME list, with shock at the beginning (top) and without (bottom). Colour-coding and labels follow the same format as in Fig. 8.

\subsection{Long-term variation of isotropy and bidirectionality}

In order to explore the solar-cycle dependence of suprathermal electron bidirectionality and isotropy, the first four panels shown in Fig. 12 represent the time variation of the percentage of isotropy (blue) and BDE (green) for both STEREOs, with moving windows of 3 months. The grey dashed lines show the mean percentage of the whole period under study. On the first two charts, the red line corresponds to the radial distance to the Sun of each s/c, while on the following two charts the green line indicates the time in hours per month of transit of ICMEs catalogued in the ICME list. The bottom panel represents the daily average sun spot number (SSN) and the average tilt angle of the HCS (Hoeksema 1995). As previously explained in Sect. 2, the energy range used for the data product has a slight difference, covering from $\sim 127 \mathrm{eV}$ to $\sim 180 \mathrm{eV}$ instead of the nominal range from $\sim 119$ to $\sim 193 \mathrm{eV}$. The two black lines of the first four panels indicate the period where this difference is present.

In spite of the small angular separation between STEREO-A and STEREO-B (less than $11^{\circ}$ ) during March-May 2007, significant discrepancies were observed in both isotropy and BDE. Some contribution of electrons escaping from the Earth's magnetosphere at STEREO-B (Opitz et al. 2014) or a shielding effect of the magnetospheric obstacle cannot be discarded as a possible source of this discrepancy at the earliest part of the plots.

The isotropy (panels 1 and 2 in Fig. 12) show periodic fluctuations, roughly coincident with the orbital period for both s/c. However, while STEREO-B tends always to observe more isotropy when it is located farther from the Sun, the origin of the periodicity for STEREO-A is unclear and not obviously correlated with the heliocentric distance, heliographic latitude, or planetary connections along the IMF (not shown in the figure). Since the amplitude of the heliocentric distance variation along its orbit is higher for STEREO-B than for STEREO-A, the recurrence observed at STEREO-B could be the result of increasing cumulative effects of scattering and/or disconnection events;

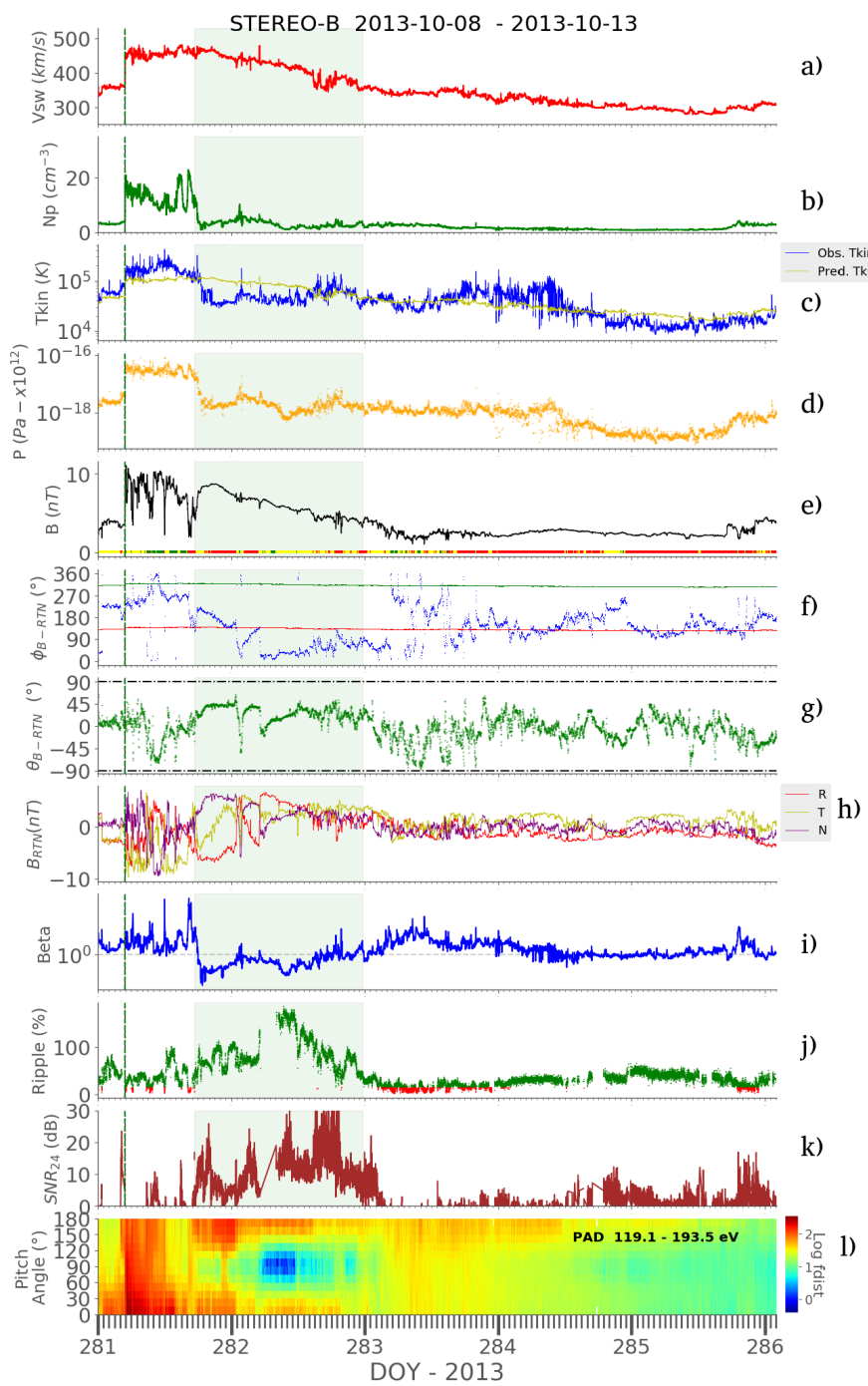

Fig. 11. STEREO-B observations during a period in October 2013 with one of the studied ICMEs. The panel content follows the same format as in Fig. 2.

however, given the narrow interval of variation of heliocentric distances covered by both s/c (below 0.087 au for STEREO-B and below 0.012 au for STEREO-A), the ultimate origin of the quasi-periodic behaviour at both s/c requires further investigation.

Apart from this periodicity, there is no obvious trend correlated with the evolution of the SSN, but a prominent increase in the isotropy during late 2009 and early 2010 was observed by both $\mathrm{s} / \mathrm{c}$, in coincidence with the quick increase in the HCS tilt angle, which marks the end of the solar minimum and the start of the rising phase of solar cycle 24 . It should be noted that suprathermal electron isotropy is frequently observed near interplanetary HCS crossings (Crooker et al. 2003), and is therefore subject to the influence of the global HCS tilt angle and the s/c latitude.

For both s/c, the isotropy is overall more frequent near solar minimum (2008-2010) than during the increasing phase of the solar cycle (2011-2014), although this tendency is weak compared with the recurrent fluctuations.

The bidirectionality (panels 3 and 4 in Fig. 12) roughly follows an increasing trend with increasing solar activity level (SSN and HCS tilt angle). This means that bidirectional periods are longer and more frequent during solar maximum than near solar 


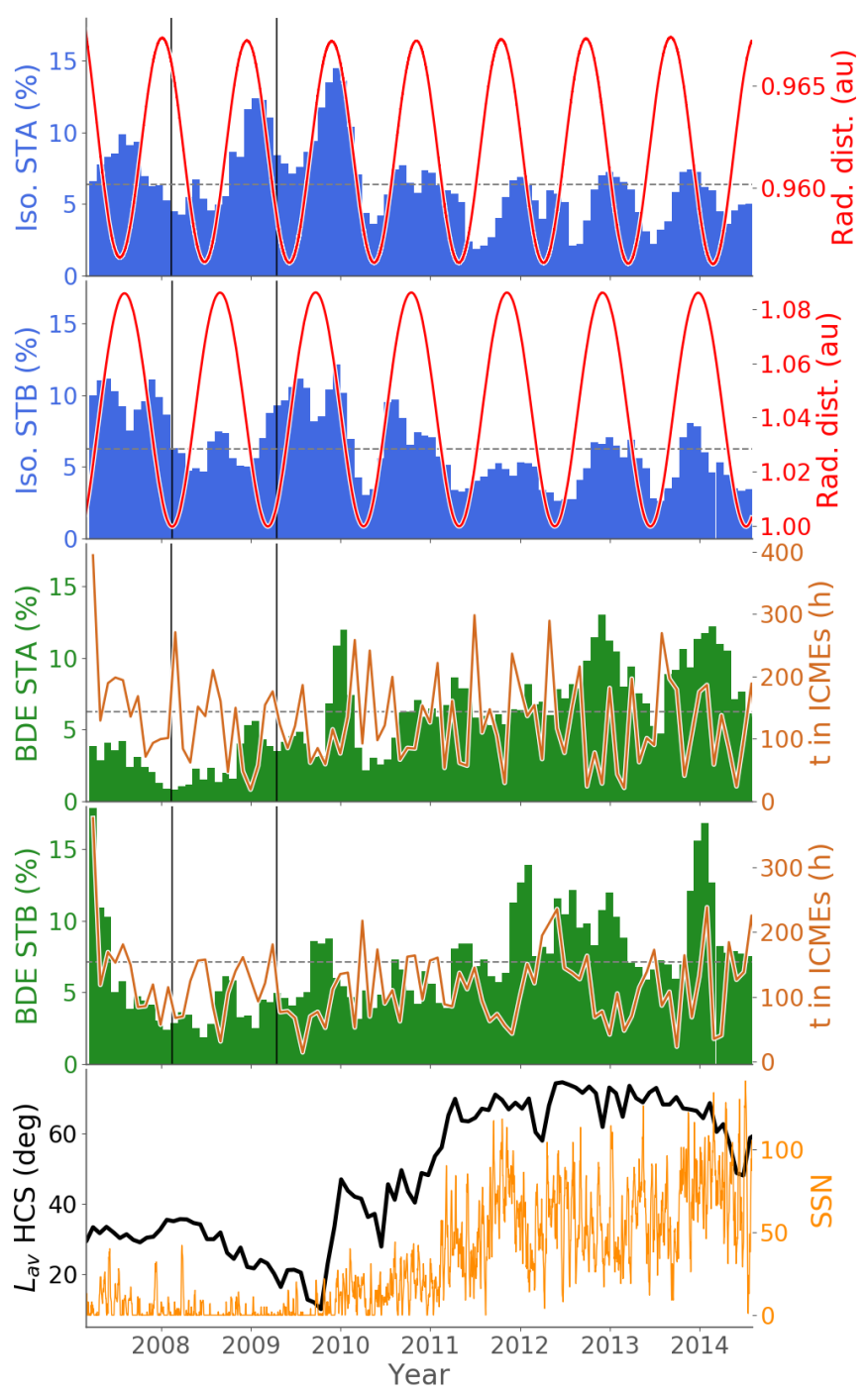

Fig. 12. Temporal variation of the time percentage during 3 months of isotropic periods (blue) and bidirectional periods (green). From top to bottom: percentages of time appearance calculated for a running window of three months of isotropy for STEREO-A, isotropy for STEREO$\mathrm{B}$, and with the heliocentric distance of both s/c overplotted; BDE for STEREO-A, BDE for STEREO-B, accompanied by the time transit of ICMEs catalogued in ICME list; finally, SSN and latitudinal angle of the HCS (see text for details).

minimum. At the same time, the fraction of time corresponding to ICME transits (solid line in panels 3 and 4) follows the same trend. This result matches what was expected, as one of the properties of the ICMEs is to present BDE (Zurbuchen \& Richardson 2006), and the ICME occurrence rate increases during solar maxima (Owens et al. 2007; Richardson \& Cane 2010; Li et al. 2018). It is also notable that STEREO-A observed a local maximum in the rate of BDE during late 2009 and early 2010, just after the quick increase in the HCS tilt angle.

\subsection{Catalogues of isotropic and bidirectional periods}

As seen in previous sections, the analyses of BDE and the isotropy provide substantial information about the topology of the IMF and the interaction processes, and about the identification of interplanetary structures such as ICMEs and HFDs.

Taking advantage of the potential of the method and criteria explained in Sects. 3.1 and 3.2.2, two different catalogues (isotropy and BDE periods) for both STEREOs have been created. These lists constitute a valuable data product for future studies of the suprathermal electrons in the SW.

The procedure for the creation of the period lists is as follows. First of all, a moving window ( $\sim 20 \mathrm{~min})$ calculates the time percentage where the imposed threshold ( $\gamma \leq \gamma_{\text {th }}$ for isotropy; or $A_{2}>0, \gamma>\gamma_{\text {th }}$ and $S / N_{24}>S / N_{\text {th }}$ for BDE) is fulfilled. If the condition is fulfilled for at least $10 \mathrm{~min}$ of that moving window, the period is a candidate for the list. When two or more candidates have a separation of less than $2 \mathrm{~h}$, they are considered to be the same event.

Four different catalogues (STEREO-A and STEREO-B for isotropic and BDE periods) are available at the CDS, and contain the following information. The beginning and the end of each period is marked (first and second column), as well as its duration (third column). Also, the column "Catalogued" shows whether the corresponding period coincides with some of the structures marked in the lists used in this work (ICME list, SIR list, or Shock list; see beginning of Sect. 3). For the shocks appearing in the Shock list, each shock is considered to affect an interval with a duration of $2 \mathrm{~h}$ centred at the time of the shock. FF represents fast-forward shocks, and FR corresponds to fastreverse shocks. The mean value of some physical parameters is provided with their standard error of the mean for the rest of the columns.

On the one hand, STEREO-A observed 2125 of isotropic periods of at least $10 \mathrm{~min}$ during the seven years of observation between March 2007 and July 2014, while STEREO-B crossed 2367 , with an average duration of the periods of $\sim 150 \mathrm{~min}$ and a mean anisotropy index $\gamma$ of $17 \%$ for both $\mathrm{s} / \mathrm{c}$. On the other hand, STEREO-A went through 1227 periods with BDE during the interval under study, while STEREO-B crossed 1333. The mean time duration is $\sim 200 \mathrm{~min}$; $S / N_{24}$ shows a mean value of $\sim 7 \mathrm{~dB}$ and the anisotropy index $\gamma, \sim 45 \%$, for both s/c as well.

\section{Summary and conclusions}

The shape of suprathermal electron PADs in the SW carries relevant information about the physical conditions at the solar source and during the interplanetary propagation, and about the largescale topology of the IMF. In particular, the presence of BDE is often a signature of closed magnetic field structures, while the presence of isotropic periods could be an indicator of disconnection from the Sun by reconnection or of enhanced interplanetary scattering.

The method presented in this work (see Sect. 3.1) is a new approach to characterise the PAD shape, and allows us not only to identify BDE and isotropy in the SW, but also to obtain the intensity, the mean flux, the anisotropy, and to categorise different types of PADs by comparing the Legendre polynomial fitting coefficients. Previous methods, such as Anderson et al. (2012), Chen et al. (2014), and Graham et al. (2017), were designed to study a specific characteristic of the PADs instead of a global characterisation of the PAD shape.

Two different thresholds $\left(\gamma_{\text {th }}\right.$ and $\left.S / N_{\text {th }}\right)$ can be used for an easy identification of isotropic and BDE periods. Isotropic periods are efficiently identified selecting those PAD with $\gamma \leq 15 \%$. The clearest BDE intervals can be selected using the combined condition $S / N_{24}>0, \gamma>15 \%$, and $A 2>0$. These cuts have been validated using extensive samples of STEREO/SWEA data during 2007-2014 (see Sects. 3.2.1 and 3.2.2). Although the selected threshold criterion is very restrictive for BDE (i.e. there are periods that could be not identified as such), establishing common criteria allows us to compare the suprathermal electrons 
PADs in relative terms. The method is well suited for automatisation, and can be used directly for space weather applications.

As reported by previous studies, anisotropy anticorrelates with the plasma $\beta$ and BDE are frequently found inside ICMEs. The $S / N_{24}$ (indicator of the symmetry of PADs) is higher for periods of BDE, and is clearly distinguishable when an average ICME is observed. The mean value of the $S / N_{24}$ for the whole period under analysis is negative, while for the ICMEs in the ICME list is positive.

Using a superposed epoch analysis for a large sample of events, the amount of BDE and isotropy during ICMEs and in the post-ICME region has been analysed. The main conclusions of this analysis are as follows:

- On average, the amount of BDE tends to gradually increase in the sheath (when it exists), reaching maximum values during the transit of the middle part of the MO. It drops at the exit of the MO and gradually decreases in the post-ICME period (defined as 1.2 times the duration of the ICME), which still shows a notable amount of BDE. The observed behaviour can be interpreted in terms of erosion by reconnection with the ambient IMF, i.e. the intervals preceding and following the MO contain some closed field lines mixed with reconnected field lines that were formerly closed. Alternatively, these BDE intervals could result from the reflection in converging lines around the ICME or at the shock or the sheath.

- Contrary to Shodhan et al. (2000), we find the observations suggest that $\mathrm{BDE}$ periods have a tendency to concentrate in the middle part of MOs, rather than being randomly distributed.

- ICMEs with shock present a higher percentage of BDE inside MOs than those without shock. The distinction between MO and sheath and post-ICME in terms of BDE is also clearer for ICMEs with shock. This behaviour suggests that slow ICMEs (those not driving a shock) show a higher degree of erosion by reconnection due to their longer transit times to $1 \mathrm{au}$.

- Isotropy is much less common inside the MO than in ambient SW, and even less far from its boundaries. This can be interpreted as a direct consequence of the smooth magnetic field (weak scattering conditions) and predominantly closed topology (not reconnected) inside the MO. On the other hand, isotropy is more common in the surroundings of the MOs (sheath and post-ICME regions) than on average SW. This is a possible indication of the presence of disconnected field lines and/or stronger scattering conditions.

The long-term behaviour of the fraction of time when the suprathermal electrons show isotropy or bidirectionality has been studied separately for STEREO-A and STEREO-B. This analysis draws the following conclusions:

- Both s/c observe recurrent fluctuations of the isotropy, coupled with the orbital periods. Periodicity at STEREO-B is apparently related to the variation of heliocentric distance, but this is not the case for STEREO-A. The ultimate origin of this periodicity is uncertain and requires further investigation.

- The isotropy is overall more frequent near solar minimum than during the increasing phase of the solar cycle, although this tendency is weak compared with the recurrent fluctuations described above.

- As expected, the BDE increases with the SSN at the same time as the number of ICMEs does. Nevertheless, the sudden change in the tilt angle of the HCS between late 2009 and early 2010 is accompanied by a large increase in the isotropy rate observed by both $\mathrm{s} / \mathrm{c}$ (which reach maximum values) and in the BDE rate observed by STEREO-A.

- Previous works (e.g. Lavraud et al. 2010) clearly show more BDE for STEREO-B than for STEREO-A. In this work, even though the defined threshold does not allow us to compare quantitatively the time-percentage of BDE, a clear tendency to observe more BDE at STEREO-B than at STEREO-A is found too. Also, since the imposed threshold is very restrictive, the time percentage is systematically lower than in the mentioned study (15-20\% versus 5-10\%).

The catalogues of isotropic and BDE periods presented in this work, and available at CDS, constitute a valuable data product for future detailed studies of the behaviour and topology of the IMF lines, apart from being a validation test for the method. They also open the way for an automatic classification of different PADs and different structures of the SW. The isotropic catalogue is useful for identifying HFDs, highly-scattered strahl, or eroded IMF lines; the BDE catalogue is useful not only for ICMEs analysis, but also for SIRs or the study of suprathermal electrons around shocks.

The method presented here can be extended (using other harmonics, their coefficients, and other $\mathrm{S} / \mathrm{N}$ calculations) to characterise other interesting PAD features (such as pancake) or their properties (e.g. width of strahl). The decomposition into harmonics is also a better approach for the application of machinelearning based classifiers, instead of the PAD itself, as the number of coefficients is half of the number of points (which is easier to compute); the relation between them also adds substantial information (rather than the relation between points of the VDF) and the sign of each provides further information of the shape of the PAD. The method has potential for automatic detection of ICMEs or their different parts, using the identification of BDE as a complement to other interplanetary signatures, as well as other interplanetary structures in the SW, with great interest also for space weather applications. An analysis of the data from future missions like Solar Orbiter or the Parker Solar Probe will also contribute to the understanding of the different PADs, and especially how the BDE and the isotropy vary with the heliocentric distance and the heliographic latitude. The method opens up new ways for a better understanding of the energy dependence of the strahl, perform statistics of strahl width, and the calculated coefficients can be correlated to other physical parameters, compared to solar energetic particle events (e.g. when HFDs occur). Furthermore, it allows us to study suprathermal electrons in other different contexts such as planetary magnetospheres.

Acknowledgements. We acknowledge the financial support by the Spanish MINECO-FPI-2016 predoctoral grant with FSE, and its project ESP2017-88436$\mathrm{R}$. We would also like to thank the different instrument teams of STEREO mission for their open-access data policy. This paper uses data from the Heliospheric Shock Database, generated and maintained at the University of Helsinki. We are very grateful to E. Sánchez-Regidor, T. Nieves-Chinchilla, L. Jian, B. Heber, A. Klassen, N. Dresing and J. Guo for all their help in this work. We also thank B. Lauvraud for the support on the usage of SWEA data. Finally, we thank the referee's labour, which substantially contributed to the improvement of this study, as well as the editor.

\section{References}

Acuña, M. H., Curtis, D., Scheifele, J. L., et al. 2008, Space Sci. Rev., 136, 203 Agueda, N., \& Lario, D. 2016, ApJ, 829, 131

Anderson, B. R., Skoug, R. M., Steinberg, J. T., \& McComas, D. J. 2012, J. Geophys. Res.: Space Phys., 117, A04107

Balogh, A. 1971, Planet. Space Sci., 19, 533

Berčič, L., Maksimović, M., Landi, S., \& Matteini, L. 2019, MNRAS, 486, 3404 Bothmer, V., Desai, M. I., Marsden, R. G., et al. 1996, A\&A, 316, 493 
Bürgi, A. 1992, in Solar Wind Seven, Proc. 3rd COSPAR Colloquium, Goslar, Germany, Sept. 16-20, 333

Che, H., \& Goldstein, M. L. 2014, ApJ, 795, L38

Chen, Y., Friedel, R. H. W., Henderson, M. G., et al. 2014, J. Geophys. Res. Space Phys., 119, 1693

Chollet, E., Skoug, R., Steinberg, J., Crooker, N., \& Giacalone, J. 2010, AIP Conf. Proc., 1216, 600

Crooker, N. U., Gosling, J. T., \& Kahler, S. W. 2002, J. Geophys. Res.: Space Phys., 107, A2

Crooker, N. U., Larson, D. E., Kahler, S. W., Lamassa, S. M., \& Spence, H. E. 2003, Geophys. Res. Lett., 30, 1619

Dahlquist, G., \& Björck, A. 2014, Numerical Methods (Dover Publications), 113

Elliott, H. A., Henney, C. J., McComas, D. J., Smith, C. W., \& Vasquez, B. J. 2012, J. Geophys. Res.: Space Phys., 117, A09102

Feldman, W. C., Asbridge, J. R., Bame, S. J., Montgomery, M. D., \& Gary, S. P. 1975, J. Geophys. Res., 80, 4181

Feldman, W. C., Skoug, R. M., Gosling, J. T., et al. 1999, Geophys. Res. Lett., 26, 2613

Feng, H. Q., Wang, J. M., Zhao, G. Q., \& Zhao, Y. 2018, ApJ, 864, 101

Fitzenreiter, R. J., Ogilvie, K. W., Chornay, D. J., \& Keller, J. 1998, Geophys. Res. Lett., 25, 249

Galvin, A. B., Kistler, L. M., Popecki, M. A., et al. 2008, Space Sci. Rev., 136, 437

Gómez-Herrero, R., Dresing, N., Klassen, A., et al. 2017, ApJ, 840, 85

Gosling, J. T. 1975, Rev. Geophys. Space Phys., 13, 1053

Gosling, J. T., Baker, D. N., Bame, S. J., et al. 1987, J. Geophys. Res.: Space Phys., 92, 8519

Gosling, J. T., Skoug, R. M., \& Feldman, W. C. 2001, Geophys. Res. Lett., 28, 4155

Graham, G. A., Rae, I. J., Owen, C. J., et al. 2017, J. Geophys. Res.: Space Phys., 122,3858

Graham, G. A., Rae, I. J., Owen, C. J., \& Walsh, A. P. 2018, ApJ, 855, 40

Gurgiolo, C., \& Goldstein, M. L. 2017, Ann. Geophys., 35, 71

Hammond, C. M., Feldman, W. C., Mccomas, D. J., Phillips, J. L., \& Forsyth, R. J. 1996, A\&A, 316, 350

Hoeksema, J. T. 1995, Space Sci. Rev., 72, 137

Horaites, K., Boldyrev, S., Wilson, L. B., Viñas, A. F., \& Merka, J. 2018 MNRAS, 474, 115

Howlett, J., Abramowitz, M., \& Stegun, I. A. 2007, Math. Gaz., 50, 358

Jian, L., Russell, C. T., Luhmann, J. G., \& Skoug, R. M. 2006a, Sol. Phys., 239, 393

Jian, L., Russell, C. T., Luhmann, J. G., \& Skoug, R. M. 2006b, Sol. Phys., 239, 337

Jian, L. K., Russell, C. T., Luhmann, J. G., Galvin, A. B., \& Simunac, K. D. 2013, AIP Conf. Proc., 1539, 191

Jian, L. K., Russell, C. T., Luhmann, J. G., \& Galvin, A. B. 2018, ApJ, 855, 114

Jian, L. K., Luhmann, J. G., Russell, C. T., \& Galvin, A. B. 2019, Sol. Phys., 294, 31

Kaiser, M. L., Kucera, T. A., Davila, J. M., et al. 2008, Space Sci. Rev., 136, 5

Kajdič, P., Kajdič, C., Lavraud, B., et al. 2014, J. Geophys. Res.: Space Phys., 119,7038

Kasper, J. C., Bale, S. D., Belcher, J. W., et al. 2019, Nature, 576, 228

Kilpua, E., Koskinen, H. E. J., \& Pulkkinen, T. I. 2017, Liv. Rev. Sol. Phys., 14,

Larson, D. E., Lin, R. P., McTiernan, J. M., et al. 1997, Geophys. Res. Lett., 24, 1911

Lavraud, B., Opitz, A., Gosling, J. T., et al. 2010, Ann. Geophys., 28, 233

Li, B., Cairns, I. H., Gosling, J. T., et al. 2016, J. Geophys. Res.: Space Phys., 121,925

Li, Y., Luhmann, J. G., \& Lynch, B. J. 2018, Sol. Phys., 293, 135

Liu, C. M., Fu, H. S., Cao, J. B., et al. 2017, Geophys. Res. Lett., 44, 116
Luhmann, J. G., Curtis, D. W., Schroeder, P., et al. 2008, Space Sci. Rev., 136, 117

Maksimovic, M., Zouganelis, I., Chaufray, J. Y., et al. 2005, J. Geophys. Res.: Space Phys., 110, A09104

McComas, D. J. 1995, Rev. Geophys., 33, 603

McComas, D. J., Gosling, J. T., Phillips, J. L., et al. 1989, J. Geophys. Res., 94, 6907

Montgomery, M. D., Asbridge, J. R., Bame, S. J., \& Feldman, W. C. 1974, J. Geophys. Res., 79, 3103

Mullan, D. J., \& Smith, C. W. 2006, Sol. Phys., 234, 325

Newbury, J. A. 1996, EOS Trans. Am. Geophys. Union, 77, 471

Newville, M., Ingargiola, A., Stensitzki, T., \& Allen, D. 2014, https://doi. org $/ 10.5281 /$ zenodo. 11813

Newville, M., Otten, R., Nelson, A., et al. 2019, https://doi.org/10.5281/ zenodo. 3381550

Nieves-Chinchilla, T., Gómez-Herrero, R., Viñas, A., et al. 2011, J. Atm. SolarTerr. Phys., 73, 1348

Opitz, A., Sauvaud, J.-A., Klassen, A., et al. 2014, J. Geophys. Res.: Space Phys., 119,6342

Owens, M. J., \& Crooker, N. U. 2006, J. Geophys. Res.: Space Phys., 111, A10104

Owens, M. J., \& Forsyth, R. J. 2013, Liv. Rev. Sol. Phys., 10, 5

Owens, M. J., Schwadron, N. A., Crooker, N. U., Hughes, W. J., \& Spence, H. E. 2007, Geophys. Res. Lett., 34, L06104

Owens, M. J., Crooker, N. U., \& Schwadron, N. A. 2008, J. Geophys. Res.: Space Phys., 113, 12103

Owens, M. J., Crooker, N. U., \& Lockwood, M. 2013, J. Geophys. Res.: Space Phys., 118, 1868

Owens, M. J., Lockwood, M., Riley, P., \& Linker, J. 2017, J. Geophys. Res.: Space Phys., 122, 980

Pagel, C., Crooker, N. U., \& Larson, D. E. 2005a, Geophys. Res. Lett., 32, 1

Pagel, C., Crooker, N. U., Larson, D. E., Kahler, S. W., \& Owens, M. J. 2005b, J. Geophys. Res.: Space Phys., 110, A01103

Pagel, C., Gary, S. P., De Koning, C. A., Skoug, R. M., \& Steinberg, J. T. 2007, J. Geophys. Res.: Space Phys., 112, A04103

Phillips, J. L., Feldman, W. C., Gosling, J. T., Hammond, C. M., \& Forsyth, R. J. 1996, AIP Conf. Proc., 382, 293

Richardson, I. G., \& Cane, H. V. 2010, Sol. Phys., 264, 189

Rodríguez-Pacheco, J., Cid, C., Blanco, J. J., \& Sequeiros, J. 2003, Sol. Phys., 213,121

Ruffenach, A., Lavraud, B., Farrugia, C. J., et al. 2015, J. Geophys. Res.: Space Phys., 120, 43

Sanderson, T. R., Marsden, R. G., Reinhard, R., Wenzel, K. P., \& Smith, E. J. 1983, Geophys. Res. Lett., 10, 916

Sanderson, T. R., Reinhard, R., van Nes, P., \& Wenzel, K.-P. 1985, J. Geophys. Res.: Space Phys., 90, 19

Sauvaud, J.-A., Larson, D., Aoustin, C., et al. 2008, Space Sci. Rev., 136, 227

Shodhan, S., Crooker, N. U., Kahler, S. W., et al. 2000, J. Geophys. Res.: Space Phys., 105, 27261

Štverák, Š., Trávníček, P., Maksimovic, M., et al. 2008, J. Geophys. Res.: Space Phys., 113, 3103

Viñas, A. F., Wong, H. K., \& Klimas, A. J. 2000, ApJ, 528, 509

Walsh, A. P., Arridge, C. S., Masters, A., et al. 2013, Geophys. Res. Lett., 40, 2495

Wang, J., Liu, Q., \& Zhao, Y. 2018, Sol. Phys., 293, 116

Winslow, R. M., Lugaz, N., Schwadron, N. A., et al. 2016, J. Geophys. Res.: Space Phys., 121, 6092

Wu, M., Lu, Q., Volwerk, M., et al. 2013, J. Geophys. Res.: Space Phys., 118, 4804

Zurbuchen, T. H., \& Richardson, I. G. 2006, Space Sci. Rev., 123, 31 
F. Carcaboso et al.: Bidirectional and isotropic periods in solar wind

\section{Appendix A: Magnetic obstacle candidates showing longer bidirectionality periods}

Table A.1. Selection of the candidates with long and clear periods of BDE observed by STEREO-A and STEREO-B.

\begin{tabular}{|c|c|c|c|}
\hline & Candidate no. & Start date & End date \\
\hline & 1 & 2008-07-05 06:34 & 2008-07-06 18:00 \\
\hline & 2 & 2009-07-11 23:10 & 2009-07-13 05:45 \\
\hline & 3 & $2009-10-1621: 35$ & $2009-10-1722: 16$ \\
\hline & 4 & 2009-11-01 08:00 & 2009-11-03 02:00 \\
\hline & 5 & 2009-12-09 09:00 & $2009-12-1023: 13$ \\
\hline & 6 & 2010-12-15 10:20 & 2010-12-16 04:00 \\
\hline & 7 & 2011-01-16 05:40 & 2011-01-17 10:10 \\
\hline & 8 & 2011-02-01 10:25 & 2011-02-02 18:40 \\
\hline & 9 & 2011-03-12 00:25 & 2011-03-12 16:47 \\
\hline & 10 & 2011-03-19 23:34 & $2011-03-2101: 30$ \\
\hline & 11 & $2011-03-2306: 55$ & 2011-03-24 23:17 \\
\hline & 12 & 2011-04-06 09:40 & 2011-04-06 23:41 \\
\hline & 13 & 2011-07-23 09:41 & 2011-07-24 11:45 \\
\hline & 14 & 2011-11-26 01:30 & $2011-11-2613: 30$ \\
\hline & 15 & 2011-11-27 00:20 & 2011-11-28 05:00 \\
\hline & 16 & $2012-01-2522: 10$ & $2012-01-2622: 00$ \\
\hline & 17 & 2012-03-04 10:34 & 2012-03-04 22:00 \\
\hline & 18 & 2012-03-17 04:10 & 2012-03-18 06:00 \\
\hline & 19 & 2012-03-20 05:00 & 2012-03-20 14:45 \\
\hline & 20 & 2012-07-11 09:10 & 2012-07-13 04:00 \\
\hline & 21 & 2012-07-16 00:45 & 2012-07-16 18:00 \\
\hline \multirow[t]{22}{*}{ STEREO-A } & 22 & 2012-07-17 03:40 & 2012-07-18 00:25 \\
\hline & 23 & 2012-10-05 16:10 & 2012-10-07 12:00 \\
\hline & 24 & $2012-10-1122: 38$ & 2012-10-13 02:30 \\
\hline & 25 & $2012-11-12$ 15:23 & 2012-11-13 15:05 \\
\hline & 26 & $2012-11-26$ 15:00 & 2012-11-28 01:22 \\
\hline & 27 & 2013-04-23 00:00 & $2013-04-23$ 19:30 \\
\hline & 28 & 2013-05-03 05:50 & 2013-05-04 16:10 \\
\hline & 29 & 2013-06-27 16:17 & 2013-06-28 00:37 \\
\hline & 30 & 2013-08-10 17:24 & 2013-08-12 07:00 \\
\hline & 31 & 2013-08-22 23:15 & $2013-08-24$ 23:25 \\
\hline & 32 & 2013-09-21 18:20 & 2013-09-22 23:20 \\
\hline & 33 & 2013-10-23 04:12 & 2013-10-24 11:37 \\
\hline & 34 & 2013-11-04 20:00 & 2013-11-06 01:30 \\
\hline & 35 & 2013-11-12 02:00 & 2013-11-13 03:00 \\
\hline & 36 & 2013-11-14 09:00 & 2013-11-14 21:00 \\
\hline & 37 & 2013-12-02 06:00 & 2013-12-04 07:40 \\
\hline & 38 & 2014-02-06 11:34 & 2014-02-07 20:30 \\
\hline & 39 & 2014-02-16 15:06 & 2014-02-17 16:15 \\
\hline & 40 & 2014-03-08 02:38 & 2014-03-08 23:12 \\
\hline & 41 & 2014-04-10 06:05 & 2014-04-10 15:35 \\
\hline & 42 & 2014-04-12 11:40 & 2014-04-12 20:33 \\
\hline & 43 & 2014-06-10 04:10 & 2014-06-10 23:50 \\
\hline
\end{tabular}

Table A.1. continued.

\begin{tabular}{|c|c|c|c|}
\hline & Candidate no. & Start date & End date \\
\hline & 44 & 2008-10-19 01:02 & 2008-10-20 11:32 \\
\hline & 45 & 2008-12-31 02:00 & 2009-01-01 07:20 \\
\hline & 46 & 2010-09-19 23:22 & 2010-09-20 06:45 \\
\hline & 47 & 2010-12-02 09:55 & 2010-12-03 11:11 \\
\hline & 48 & 2011-01-18 00:00 & 2011-01-18 09:38 \\
\hline & 48 & 2011-03-07 19:10 & 2011-03-08 17:21 \\
\hline & 49 & 2011-03-11 15:18 & 2011-03-12 12:00 \\
\hline & 50 & 2011-04-01 04:00 & 2011-04-01 13:50 \\
\hline & 50 & 2011-06-01 17:35 & 2011-06-02 18:00 \\
\hline & 51 & 2011-06-17 07:40 & 2011-06-18 04:00 \\
\hline & 52 & 2011-10-04 02:00 & 2011-10-04 12:40 \\
\hline & 53 & 2011-12-02 16:03 & 2011-12-03 11:00 \\
\hline & 54 & 2012-01-11 17:43 & 2012-01-12 13:42 \\
\hline & 55 & 2012-01-17 07:30 & 2012-01-18 09:00 \\
\hline & 56 & 2012-01-19 01:46 & 2012-01-19 16:43 \\
\hline & 57 & 2012-03-09 01:00 & 2012-03-09 11:30 \\
\hline & 58 & 2012-03-11 01:34 & 2012-03-12 21:51 \\
\hline & 59 & 2012-03-30 01:37 & 2012-03-30 08:30 \\
\hline & 60 & 2012-04-17 09:40 & 2012-04-18 09:05 \\
\hline & 61 & 2012-05-09 13:30 & 2012-05-10 10:40 \\
\hline & 62 & 2012-05-13 03:00 & 2012-05-14 04:30 \\
\hline \multirow[t]{25}{*}{ STEREO-B } & 63 & 2012-06-18 23:47 & 2012-06-20 10:00 \\
\hline & 64 & 2012-07-04 11:40 & 2012-07-05 12:50 \\
\hline & 65 & 2012-07-24 20:00 & $2012-07-25$ 12:00 \\
\hline & 66 & 2012-09-04 05:30 & 2012-09-05 20:58 \\
\hline & 67 & $2012-09-2323: 38$ & 2012-09-24 09:46 \\
\hline & 68 & $2012-10-2604: 00$ & 2012-10-27 10:00 \\
\hline & 69 & 2012-11-04 01:22 & 2012-11-05 18:00 \\
\hline & 70 & 2012-11-20 02:00 & 2012-11-20 12:40 \\
\hline & 71 & 2012-11-28 07:37 & 2012-11-29 07:00 \\
\hline & 72 & 2013-03-08 08:00 & 2013-03-10 08:45 \\
\hline & 73 & 2013-04-09 23:40 & 2013-04-10 14:00 \\
\hline & 74 & 2013-05-04 15:00 & 2013-05-06 09:40 \\
\hline & 75 & 2013-06-02 12:15 & 2013-06-06 08:52 \\
\hline & 76 & 2013-07-05 07:04 & 2013-07-07 01:47 \\
\hline & 77 & 2013-08-22 13:00 & 2013-08-23 07:00 \\
\hline & 78 & 2013-09-16 04:20 & 2013-09-17 00:25 \\
\hline & 79 & 2013-10-08 17:25 & 2013-10-09 23:35 \\
\hline & 80 & 2013-11-05 02:43 & 2013-11-05 23:47 \\
\hline & 81 & 2013-11-06 13:38 & 2013-11-07 14:00 \\
\hline & 82 & 2013-12-18 02:05 & $2013-12-2015: 00$ \\
\hline & 83 & 2013-12-21 08:25 & 2013-12-22 00:00 \\
\hline & 84 & 2013-12-29 04:12 & 2013-12-30 14:00 \\
\hline & 85 & 2014-04-01 04:53 & 2014-04-01 21:37 \\
\hline & 86 & 2014-05-08 21:00 & 2014-05-10 08:23 \\
\hline & 87 & 2014-06-10 00:00 & 2014-06-10 17:10 \\
\hline
\end{tabular}

